\title{
Siegel modular forms and finite
}

\section{symplectic groups}

\author{
Francesco Dalla Piazza ${ }^{1}$ and Bert van Geemen ${ }^{2}$
}

${ }^{1}$ Dipartimento di Scienze Fisiche e Matematiche, Università dell'Insubria, Via Valleggio 11, I-22100 Como, Italia

f .dallapiazza@uninsubria.it

${ }^{2}$ Dipartimento di Matematica, Università di Milano, Via Saldini 50, I-20133 Milano, Italia

geemen@mat .unimi.it

\begin{abstract}
The finite symplectic group $\operatorname{Sp}(2 g)$ over the field of two elements has a natural representation on the vector space of Siegel modular forms of given weight for the principal congruence subgroup of level two. In this paper we decompose this representation, for various (small) values of the genus and the level, into irreducible representations. As a consequence we obtain uniqueness results for certain modular forms related to the superstring measure, a better understanding of certain modular forms in genus three studied by D'Hoker and Phong as well as a new construction of Miyawaki's cusp form of weight twelve in genus three.
\end{abstract}

\section{Introduction}

The vector spaces of Siegel modular forms for the principal congruence subgroup $\Gamma_{g}(n)$ of level $n$ are of great interest in various areas in mathematics. For example in geometry, where they correspond to holomorphic tensors

e-print archive: http://arXiv.org/abs/hep-th/0804.3769 
on moduli spaces of abelian varieties, in arithmetic, by way of the L-series of Hecke eigenforms and their relation to Galois representations, and in mathematical physics, where they come up in the bosonic and superstring measures. As the dimension of these spaces grows fast with both genus $g$ and level $n$ it is convenient to use the natural representation of the finite symplectic group $\Gamma_{g} / \Gamma_{g}(n)$ on these spaces. This allows one to pick out, in a canonical fashion, subspaces, i.e., subrepresentations, which can then be analyzed more thoroughly.

In this paper we will be concerned only with the case $n=2$ and the quotient group $\mathrm{Sp}(2 g)$, the finite symplectic group over the field of two elements. For genus $g \leq 3$ the spaces of modular forms on $\Gamma_{g}(2)$ of even weight have an explicit description in terms of theta series and this allows us to determine the corresponding representations explicitly for small $k$, usually with the help of a computer. In general, the theta series generate only a subspace of the space of all modular forms, but as this subspace is an $\operatorname{Sp}(2 g)$-subrepresentation, it is still interesting to find its decomposition.

As an application of our results we prove in Section 7 some uniqueness results on the modular forms $\Xi_{8}\left[0^{(g)}\right]$ for small $g$. These modular forms are of considerable interest in superstring theory (see [19]). We also investigate the space spanned by the 336 modular forms of weight 6 and genus three studied extensively by D'Hoker and Phong in [8]. We show that this space has dimension 105, that the $\operatorname{Sp}(6)$-representation on it is irreducible and we identify the representation in the character table of Frame [9]. Moreover, any function in this space turns out to be a cusp form and the sum of the squares of the 336 functions is the cusp form $F_{12}$ found by Miyawaki whose Spinor L-function was recently determined by B. Heim.

In Appendix B (Section B) we recall the basics on the transformation theory of the theta functions which we used in order to determine the representations of $\operatorname{Sp}(2 g)$ on modular forms.

\section{Siegel modular forms of level two}

\subsection{Introduction}

In this section we recall the action of a finite symplectic group on the modular forms of a given weight $k$ for the congruence subgroup of $\Gamma_{g}(n)$ of $\operatorname{Sp}(2 g, \mathbf{Z})$ in the case $n=2$ (for other positive integers one has a similar action). 


\subsection{The groups $\Gamma_{g}, \Gamma_{g}(n)$ and $\operatorname{Sp}(2 g)$}

We will write $\Gamma_{g}:=\operatorname{Sp}(2 g, \mathbf{Z})$ and

$$
\Gamma_{g}(n):=\{M \in \operatorname{Sp}(2 g, \mathbf{Z}): M \equiv I \bmod n\},
$$

is a normal subgroup. In case $n=2$, we have

$$
\Gamma_{g}(2)=\operatorname{ker}\left(\operatorname{Sp}(2 g, \mathbf{Z}) \longrightarrow \operatorname{Sp}(2 g):=\operatorname{Sp}\left(2 g, \mathbf{F}_{2}\right)\right),
$$

where we write $\mathbf{F}_{2}:=\mathbf{Z} / 2 \mathbf{Z}$ for the field with two elements. The reduction mod two map is surjective [16, V.6 Lemma 25] and thus we have an isomorphism

$$
\operatorname{Sp}(2 g) \cong \Gamma_{g} / \Gamma_{g}(2) .
$$

The order of this group is (cf. [18, Theorem 6.18]):

$$
\begin{aligned}
|\operatorname{Sp}(2 g)| & =2^{2 g-1}\left(2^{2 g}-1\right)|\operatorname{Sp}(2 g-2)| \\
& =2^{2 g-1}\left(2^{2 g}-1\right) 2^{2 g-3}\left(2^{2 g-2}-1\right) \cdots 2\left(2^{2}-1\right) .
\end{aligned}
$$

In particular, $|\operatorname{Sp}(2 g)|=6,6 !=720,36 \cdot(8 !)$ for $g=1,2,3$.

The group $\operatorname{Sp}(2 g)$ acts (linearly) on the $2^{2 g}$ points of $\mathbf{F}_{2}^{2 g}$ (sometimes called period characteristics) and it acts non-linearly on the (theta) characteristics $\left[\begin{array}{l}a_{1} \ldots a_{g} \\ b_{1} \ldots b_{g}\end{array}\right]$ with $a_{i}, b_{i} \in\{0,1\}$, see Appendix A. It has two orbits on the characteristics, of length $2^{g-1}\left(2^{g}+1\right)$ and $2^{g-1}\left(2^{g}-1\right)$ respectively, the first orbit consists of the even characteristics which are those with $\sum a_{i} b_{i} \equiv 0$ mod 2, the other orbit are the odd characteristics.

One has the following description of $\operatorname{Sp}(2 g)$ for small $g$ : the action of $\operatorname{Sp}(2)$ on the three non-zero points in $\mathbf{F}_{2}^{2}-\{0\}$ induces an isomorphism $\operatorname{Sp}(2)=\operatorname{SL}\left(2, \mathbf{F}_{2}\right) \cong S_{3}$, the action of $\Gamma_{4}$ on the six odd characteristics (for $g=2)$ induces an isomorphism $\operatorname{Sp}(4) \cong S_{6}$, and $\operatorname{Sp}(6)$ is isomorphic to the subgroup of elements with determinant 1 of the Weyl group $W\left(E_{7}\right)$ in its standard seven-dimensional representation (A.4, [1] where $\mathrm{Sp}(6)=S_{6}(2)$ ).

\subsection{The subgroups $\mathrm{O}^{+}(2 g)$ and $\mathrm{O}^{-}(2 g)$ of $\operatorname{Sp}(2 g)$}

The group $\Gamma_{g}$ acts on $V=\mathbf{F}_{2}^{2 g}$, and thus on the characteristics, through its quotient $\operatorname{Sp}(2 g)=\Gamma_{g} / \Gamma_{g}(2)$. The stabiliser subgroup of the even characteristic $[0]:=\left[\begin{array}{l}0 \ldots . .0 \\ 0 . .0\end{array}\right]$ is the subgroup $\Gamma_{g}(1,2)$, where we define more generally 
subgroups (cf. [16, V.2, p.178]):

$$
\Gamma_{g}(n, 2 n):=\left\{M \in \Gamma_{g}(n): \operatorname{diag} A^{t} B \equiv \operatorname{diag} C^{t} D \equiv 0 \bmod 2 n\right\} .
$$

In case $n$ is even, $\Gamma_{g}(n, 2 n)$ is a normal subgroup of $\Gamma_{g}(1)$.

The image of $\Gamma_{g}(1,2)$ in $\operatorname{Sp}(2 g)$ is denoted by $O^{+}(2 g)$ :

$$
O^{+}(2 g):=\Gamma_{g}(1,2) / \Gamma_{g}(2) \quad(\subset \operatorname{Sp}(2 g)) .
$$

As $\operatorname{Sp}(2 g)$ acts transitively on the even theta characteristics, there is a natural bijection

$$
\mathrm{Sp}(2 g) / O^{+}(2 g) \longrightarrow\{\Delta: \Delta \text { even }\}, \quad h O^{+}(2 g) \longmapsto h \cdot[0] .
$$

In particular, $\left[\operatorname{Sp}(2 g): O^{+}(2 g)\right]=2^{g-1}\left(2^{g}+1\right)$. One has $O^{+}(2) \cong \mathbf{Z} / 2 \mathbf{Z}$ and $\mathrm{O}^{+}(4)$ is isomorphic to the subgroup of the symmetric group $S_{6} \cong \operatorname{Sp}(4)$ of permutations $\sigma$ such that $\sigma(\{1,2,3\}) \subset\{1,2,3\}$ or $\sigma(\{1,2,3\})=\{4,5,6\}$, thus $S_{3} \times S_{3}$ is a subgroup of index two in $O^{+}(4)$ and $\left|O^{+}(4)\right|=(3 !) \cdot(3 !)$. $2=72$. One has $O^{+}(6) \cong S_{8}$, the symmetric group of order 8 ! and $O^{+}(8)$ is the quotient of the subgroup of elements of $W\left(E_{8}\right)$ with determinant +1 in the standard eight-dimensional representation, by the center, generated by $-I$.

Similarly, we define a subgroup $O^{-}(2 g) \subset \mathrm{Sp}(2 g)$ as the stabilizer of the odd characteristic $\left[\begin{array}{l}10 \ldots 0 \\ 10 \ldots 0\end{array}\right]$ and we have $\left[\operatorname{Sp}(2 g): O^{+}(2 g)\right]=2^{g-1}\left(2^{g}-1\right)$. One has $O^{-}(2) \cong \operatorname{Sp}(2) \cong S_{3}, O^{-}(4) \cong S_{5}$, a symmetric group, and $O^{-}(6) \cong$ $W\left(E_{6}\right)$, the Weyl group of the root system $E_{6}$.

\subsection{Modular forms of level 2}

A Siegel modular form of genus $g$, weight $k$ and level 2 is a holomorphic function on the Siegel upper half-space of genus $g$

$$
f: \mathbf{H}_{g} \longrightarrow \mathbf{C}, \quad f(M \cdot \tau)=\operatorname{det}(C \tau+D)^{k} f(\tau) \quad \forall M \in \Gamma_{g}(2)
$$

(and in case $g=1$ one should also impose a growth condition on $f$ ). Here, as usual, the action of $\Gamma_{g}$ on the Siegel upper half-space is given by

$$
M \cdot \tau:=(A \tau+B)(C \tau+D)^{-1} \quad M:=\left(\begin{array}{cc}
A & B \\
C & D
\end{array}\right) \in \operatorname{Sp}(2 g, \mathbf{Z}), \quad \tau \in \mathbf{H}_{g}
$$

The Siegel modular forms of genus $g$, weight $k$ and level 2 form a finitedimensional complex vector space denoted by $M_{k}\left(\Gamma_{g}(2)\right)$. The finite group 
$\mathrm{Sp}(2 g)$ has a representation

$$
\rho=\rho_{k}: \mathrm{Sp}(2 g) \longrightarrow \mathrm{GL}\left(M_{k}\left(\Gamma_{g}(2)\right)\right)
$$

on this vector space defined by

$$
\left(\rho\left(g^{-1}\right) f\right)(\tau):=\operatorname{det}(C \tau+D)^{-k} f(M \cdot \tau),
$$

where $M \in \Gamma_{g}$ is a representative of $g \in \operatorname{Sp}(2 g)$ and $f \in M_{k}\left(\Gamma_{g}(2)\right)$ (note that $\operatorname{det}(C \tau+D)^{-k} f(M \cdot \tau)=f(\tau)$ for $M \in \Gamma_{g}(2)$, thus the action of $M \in \Gamma_{g}$ factors over $\left.\Gamma_{g} / \Gamma_{g}(2)=\operatorname{Sp}(2 g)\right)$. The equality $\rho(g h)=\rho(g) \rho(h)$ for $g, h \in \mathrm{Sp}(2 g)$ follows from $(M N) \cdot \tau=M \cdot(N \cdot \tau)$ and $\gamma(M N, \tau)=$ $\gamma(M, N \cdot \tau) \gamma(N, \tau)$ where $\gamma(M, \tau):=\operatorname{det}(C \tau+D)$.

\subsection{The theta constants $\Theta[\sigma]$}

To determine the modular forms of even weight on $\Gamma_{g}(2)$ it is convenient to define the $2^{g}$ theta constants (see Appendix A.2):

$$
\Theta[\sigma](\tau):=\theta\left[\begin{array}{l}
\sigma \\
0
\end{array}\right](2 \tau, 0), \quad[\sigma]=\left[\begin{array}{llll}
\sigma_{1} & \sigma_{2} & \ldots & \sigma_{g}
\end{array}\right], \sigma_{i} \in\{0,1\}, \tau \in \mathbf{H}_{g} .
$$

These theta constants are "modular forms of weight $1 / 2$ " on the normal subgroup $\Gamma_{g}(2,4) \subset \Gamma_{g}(2)$, defined in 2.3 .

Basically, the invariants of degree $4 k$ of the quotient group $\Gamma_{g}(2) / \Gamma_{g}(2,4) \cong \mathbf{F}_{2}^{2 g}$ in the ring of polynomials in the $\Theta[\sigma]$ 's are modular forms of weight $2 k$ on $\Gamma_{g}(2)$. Due to the half integral weight, the quotient group $\Gamma_{g}(2) / \Gamma_{g}(2,4)$ does not actually act on the $\Theta[\sigma]$, but a central extension, the Heisenberg group $H_{g}$ does (see Appendix B) and we have to take the invariants for this Heisenberg group. The subspace of $M_{2 k}\left(\Gamma_{g}(2)\right)$ of these Heisenberg invariants is denoted by

$$
M_{2 k}^{\theta}\left(\Gamma_{g}(2)\right) \subset M_{2 k}\left(\Gamma_{g}(2)\right), \quad M_{2 k}^{\theta}\left(\Gamma_{g}(2)\right):=\mathbf{C}[\ldots, \Theta[\sigma], \ldots]_{4 k}^{H_{g}},
$$

where $\mathbf{C}[\ldots, \Theta[\sigma], \ldots]_{4 k}$ is the subspace of homogeneous polynomials of degree $4 k$ in the $\Theta[\sigma]$ 's.

\subsection{The Heisenberg group $\boldsymbol{H}_{g}$}

We define a finite (Heisenberg) group by

$$
H_{g}=\mu_{4} \times \mathbf{F}_{2}^{g} \times \mathbf{F}_{2}^{g}, \quad(s, x, u)(t, y, v)=\left(s t(-1)^{u y}, x+y, u+v\right),
$$


where $\mu_{4}=\left\{z \in \mathbf{C}: z^{4}=1\right\}$ is the multiplicative group of fourth roots of unity, and $u y=\sum u_{i} y_{i}$ for $u=\left(u_{1}, \ldots, u_{g}\right), y=\left(y_{1}, \ldots, y_{g}\right) \in \mathbf{F}_{2}^{g}$. The center of $H_{g}$ is $\mu_{4}$ and the quotient $H_{g} / \mu_{4}$ is isomorphic to $\mathbf{F}_{2}^{2 g}$.

Next, we define the (Schrödinger) representation of $H_{g}$ on polynomials in the $2^{g}$ variables $X_{\sigma}$, where $[\sigma]=\left[\sigma_{1} \sigma_{2} \ldots \sigma_{g}\right], \sigma_{i} \in\{0,1\}$ :

$$
(s, x, u) X_{\sigma}:=s(-1)^{(x+\sigma) u} X_{x+\sigma},
$$

where we now consider $\sigma_{i} \in \mathbf{F}_{2}=\{0,1\}$, so $x+\sigma=\left[x_{1}+\sigma_{1}, \ldots\right]$. This action is extended in the obvious way to polynomials in the $X_{\sigma}$ 's. The action of $H_{g}$ on $\mathbf{C}\left[\ldots, X_{\sigma}, \ldots\right]$ induces the one on $\mathbf{C}[\ldots, \Theta[\sigma], \ldots]$ under the map $X_{\sigma} \mapsto \Theta[\sigma]$, (cf. Appendix B).

\subsection{Heisenberg invariants}

Note that a homogeneous polynomial in the $X_{\sigma}$ is invariant under the subgroup $\mu_{4}$ of $H_{g}$ iff its degree is a multiple of 4 , so $H_{g}$-invariant polynomials are modular forms of even weight.

The subring of Heisenberg invariant polynomials in $\mathbf{C}\left[\ldots, X_{\sigma}, \ldots\right]$ will be denoted by $\mathbf{C}\left[\ldots, X_{\sigma}, \ldots\right]^{H_{g}}$. In Section 2.8 we give a formula for the dimension of the vector space of Heisenberg invariant polynomials of degree $4 n$.

It is not hard to construct a basis of this vector space. Each monomial in a Heisenberg invariant polynomial $P$ is a product $\prod_{i=1}^{4 n} X_{\sigma_{i}}$ which must be invariant under the action of the $(1,0, u) \in H_{g}$, hence $\sum \sigma_{i}=0$. As $P$ is also invariant under the $(1, x, 0)$, all monomials of the type $\prod_{i=1}^{4 n} X_{\sigma_{i}+x}$, for any $x \in \mathbf{F}_{2}^{g}$, have the same coefficient in $P$. Thus a basis is obtained by the $\sum_{x} \prod_{i=1}^{4 n} X_{\sigma_{i}+x}$, where $\sum \sigma_{i}=0$.

\subsection{A dimension formula}

We give a formula for the dimension of the Heisenberg invariants in the vector space $\mathbf{C}\left[\ldots, X_{\sigma}, \ldots\right]_{n}$ of homogeneous polynomials of degree $n$ in the $X_{\sigma}$ 's. Let, for fixed $g$,

$$
\rho_{n}: H_{g} \longrightarrow \mathrm{GL}\left(\mathbf{C}\left[\ldots, X_{\sigma}, \ldots\right]_{n}\right)
$$

be the representation of $H_{g}$ on this vector space. Then the space of Heisenberg invariants is the trivial subrepresentation of $\rho_{n}$, so its 
dimension is

$$
\operatorname{dim} \mathbf{C}\left[\ldots, X_{\sigma}, \ldots\right]_{n}^{H_{g}}=\left\langle\rho_{n}, 1_{H_{g}}\right\rangle_{H_{g}}
$$

the multiplicity of the trivial representation $1_{H_{g}}$ of $H_{g}$ in $\rho_{n}$. This scalar product of characters is given by

$$
\left\langle\rho_{n}, 1_{H_{g}}\right\rangle_{H_{g}}=\frac{1}{\left|H_{g}\right|} \sum_{h \in H_{g}} \operatorname{tr}\left(\rho_{n}(h)\right)
$$

where tr is the trace.

Note that if $x u=0$, but $(x, u) \neq(0,0),(1, x, u)$ has order two in $H_{g}$ and the eigenvalues of $(t, x, u) \in H_{g}$ on $\mathbf{C}\left[\ldots, X_{\sigma}, \ldots\right]_{1}$ are $t$ and $-t$, each with multiplicity $2^{g-1}$ for all $t \in \mu_{4}$. In case $x u=1,(1, x, u)$ has order four and $(t, x, u)$ has eigenvalues it and $-i t$ (with $i^{2}=-1$ ), each with multiplicity $2^{g-1}$. So if $(x, u) \neq(0,0)$ the eigenvalues of $\rho_{1}(t, x, u)$, with $t \in \mu_{4}$, are $i^{a},-i^{a}$ with $a=1$ or $a=2$, each with multiplicity $2^{g-1}$.

If $\alpha_{1}, \ldots, \alpha_{N}, N=2^{g}$ are the eigenvalues of $(t, x, u)$ on $\mathbf{C}\left[\ldots, X_{\sigma}, \ldots\right]_{1}$, the eigenvalues on $\mathbf{C}\left[\ldots, X_{\sigma}, \ldots\right]_{n}$ are the $\alpha_{1}^{m_{1}} \alpha_{2}^{m_{2}} \ldots \alpha_{N}^{m_{N}}$ with $\sum m_{i}=n$. As the trace is the sum of the eigenvalues, we get (with a variable $X$ )

$$
\sum_{n} \operatorname{tr}\left(\rho_{n}(t, x, u)\right) X^{n}=\prod_{i=1}^{N}\left(1-\alpha_{i} X\right)^{-1}
$$

So if $(x, u) \neq(0,0)$ we have

$$
\sum_{n} \operatorname{tr}\left(\rho_{n}(t, x, u)\right) X^{n}=\left(1-i^{2 a} X^{2}\right)^{-2^{g-1}}=\sum_{m}(-1)^{a m}\left(\begin{array}{c}
2^{g-1}+m-1 \\
m
\end{array}\right) X^{2 m},
$$

and in case $(x, u)=(0,0)$ the trace is just

$$
\begin{aligned}
\sum_{n} \operatorname{tr}\left(\rho_{n}(t, 0,0)\right) X^{n} & =\sum_{n} t^{n}\left(\operatorname{dim} \mathbf{C}\left[\ldots, X_{\sigma}, \ldots\right]_{n}\right) X^{n} \\
& =\sum_{n} t^{n}\left(\begin{array}{c}
2^{g}+n-1 \\
n
\end{array}\right) X^{n} .
\end{aligned}
$$

This leads to the formula (note that there are non-trivial invariants only if $n$ is a multiple of 4 )

$$
\begin{aligned}
\operatorname{dim} & \left(\mathbf{C}\left[\ldots, X_{\sigma}, \ldots\right]_{4 n}\right)^{H_{g}} \\
& =2^{-2 g}\left(\left(\begin{array}{c}
2^{g}+4 n-1 \\
4 n
\end{array}\right)+\left(2^{2 g}-1\right)\left(\begin{array}{c}
2^{g-1}+2 n-1 \\
2 n
\end{array}\right)\right) .
\end{aligned}
$$


For small $g$ we list some of these dimensions in the table below.

\begin{tabular}{lrrrr}
\hline \multicolumn{5}{c}{ Degree } \\
\hline$g$ & 4 & \multicolumn{1}{c}{8} & \multicolumn{1}{c}{12} & \multicolumn{1}{c}{16} \\
\hline 1 & 2 & 3 & 4 & 5 \\
2 & 5 & 15 & 35 & 69 \\
3 & 15 & 135 & 870 & 3993 \\
4 & 51 & 2244 & 69,615 & $1,180,395$ \\
\hline
\end{tabular}

In case $n=1$, the dimension formula gives

$$
\operatorname{dim}\left(\mathbf{C}\left[\ldots, X_{\sigma}, \ldots\right]_{4}\right)^{H_{g}}=\left(2^{g}+1\right)\left(2^{g-1}+1\right) / 3 .
$$

\subsection{The ring of modular forms}

The vector spaces $M_{2 k}^{\theta}\left(\Gamma_{g}(2)\right)$ are images of the spaces of $H_{g}$ invariants of degree $4 k$ under the surjective maps

$$
\mathbf{C}\left[\ldots, X_{\sigma}, \ldots\right]_{4 k}^{H_{g}} \longrightarrow M_{2 k}^{\theta}\left(\Gamma_{g}(2)\right):=\mathbf{C}[\ldots, \Theta[\sigma], \ldots]_{4 k}^{H_{g}}, \quad X_{\sigma} \longmapsto \Theta[\sigma] .
$$

These maps define a surjective $\mathbf{C}$-algebra homomorphism

$$
\mathbf{C}\left[\ldots, X_{\sigma}, \ldots\right]^{H_{g}} \longrightarrow M^{\theta}\left(\Gamma_{g}(2)\right):=\oplus_{k} M_{2 k}^{\theta}\left(\Gamma_{g}(2)\right)
$$

whose kernel is the ideal of algebraic relations between the $\Theta[\sigma]$ 's. So a polynomial $F\left(\ldots, X_{\sigma}, \ldots\right)$ maps to zero iff $F(\ldots, \Theta[\sigma](\tau), \ldots)=0$ for all $\tau \in \mathbf{H}_{g}$. In geometrical terms, a homogeneous polynomial $F$ lies in the kernel iff the image of $\mathbf{H}_{g}$ under the map

$$
\mathbf{H}_{g} \longrightarrow \mathbf{P}^{2^{g}-1}, \quad \tau \longmapsto(\ldots: \Theta[\sigma](\tau): \ldots)
$$

lies in the zero locus $Z(F) \subset \mathbf{P}^{2^{g}-1}$. We will describe this map for $g=$ $1,2,3$, in particular the image of the map contains an open subset for $g=$ 1,2 , thus there are no polynomials vanishing on the image. In case $g=3$ the image is a (Zariski) open subset of a hypersurface $Z\left(F_{16}\right) \subset \mathbf{P}^{7}$, for a certain homogeneous polynomial $F_{16}$ in eight variables, hence $M^{\theta}\left(\Gamma_{g}(2)\right) \cong$ $\mathbf{C}\left[\ldots, X_{\sigma}, \ldots\right]^{H_{g}} /\left(F_{16}\right)$.

For any $g, g \geq 4$, there must be (many) algebraic relations between the $\Theta[\sigma]$ 's because $\operatorname{dim} \mathbf{H}_{g}=g(g+1) / 2<2^{g}-1$, but a complete description of these relations is not known. 
The graded ring of modular forms of even weight on $\Gamma_{g}(2)$ is the normalization of the ring of the $\Theta[\sigma]$ 's (cf. [25, Theorem 2, [23, 24]]):

$$
\underset{k=0}{\infty} M_{2 k}\left(\Gamma_{g}(2)\right)=\left(\mathbf{C}[\ldots, \Theta[\sigma], \ldots]^{H_{g}}\right)^{\text {Nor }} .
$$

In case $g=1,2$ there are no relations and the ring of invariants is already normal. In case $g=3$, there is one relation $F_{16}(\ldots, \Theta[\sigma], \ldots)=0$ homogeneous of degree 16 , and Runge $[23,24]$ showed that the quotient of the ring of invariants by the ideal generated by this relation is again normal. This implies that any modular form of weight $2 k$ can be written as a homogeneous polynomial of degree $4 k$ in the $\Theta[\sigma]$ 's if $g \leq 3$, thus

$$
M_{2 k}^{\theta}\left(\Gamma_{g}(2)\right)=M_{2 k}\left(\Gamma_{g}(2)\right) \quad \text { for } \quad g=1,2,3 .
$$

This polynomial is unique for $g \leq 2$. For $g=3$ it is unique if its degree is less than 15 and else it is unique up to the addition of $F_{16} G_{4 k-16}$ where $G_{4 k-16}$ is any homogeneous polynomial of degree $4 k-16$ in the $\Theta[\sigma]$ 's.

For $g>3$ there will always be non-trivial relations and if $g>4$ the ring $\mathbf{C}[\ldots, \Theta[\sigma], \ldots]^{H_{g}}$ 's is not normal, (cf. [22, Theorem 6], but note that our $H_{g}$ is different from theirs). In case the ring is not normal, there are also quotients $G_{4 k+d} / H_{d}$ of homogeneous polynomials in the $\Theta[\sigma]$ 's, of degree $4 k+d$ and $d$, respectively, which are modular forms of weight $4 k$ (but which cannot be written as a polynomial in the $\Theta[\sigma]$ 's).

\section{Classical theta constants and representations}

\subsection{Introduction}

To describe the spaces of modular forms $M_{2 k}^{\theta}\left(\Gamma_{g}(2)\right)$ it is convenient to use also the classical theta functions with ("half integral") characteristics $\theta[\Delta]$. In particular, we are interested in decomposing these spaces into irreducible representations for the group $\operatorname{Sp}(2 g)$ and we want to describe the subspace of $\mathrm{O}^{+}$-invariants as well as $\mathrm{O}^{+}$-anti-invariants (cf. Section 3.4), these will have applications to the superstring measures.

\subsection{The quadratic relations between the $\theta[\Delta]$ 's and the $\Theta[\sigma]$ 's}

A classical formula for theta functions shows that any product of two $\Theta[\sigma]$ 's is a linear combination of the $\theta[\Delta]^{2}$. Note that there are $2^{g}$ functions $\Theta[\sigma]$ 
and thus there are $\left(2^{g}+1\right) 2^{g} / 2=2^{g-1}\left(2^{g}+1\right)$ products $\Theta[\sigma] \Theta\left[\sigma^{\prime}\right]$. This is also the number of even characteristics, and the products $\Theta[\sigma] \Theta\left[\sigma^{\prime}\right]$ span the same space (of modular forms of weight 1 ) as the $\theta[\Delta]^{2}$ 's, which has dimension $2^{g-1}\left(2^{g}+1\right)$.

As the degree of an $H_{g}$-invariant homogeneous polynomial in the $\Theta[\sigma]$ is a multiple of four, say $4 k$, it can be written as a homogeneous polynomial of degree $2 k$ in the $\theta[\Delta]^{2}$ 's. Thus for $g \leq 3$, any element in $M_{2 k}\left(\Gamma_{g}(2)\right)$ is a homogeneous polynomial of degree $2 k$ in the $\theta[\Delta]^{2}$ 's.

The $\theta[\Delta]^{2}$ are the better known functions and their transformation under $\Gamma_{g}(1)$ is easy to understand, but the $\Theta[\sigma]$ have the advantage that they are algebraically independent for $g \leq 2$ and there is a unique relation of degree 16 for $g=3$. In contrast, there are many quadratic relations between the $\theta[\Delta]^{2}$ 's, for example Jacobi's relation in $g=1$.

\subsection{A classical formula}

The classical formula used here is (cf. [16, IV.1]):

$$
\theta\left[\begin{array}{l}
a \\
b
\end{array}\right]^{2}=\sum_{\sigma}(-1)^{\sigma b} \Theta[\sigma] \Theta[\sigma+a],
$$

where we sum over the $2^{g}$ vectors $\sigma \in \mathbf{F}_{2}^{g}$ and $\left[\begin{array}{l}a \\ b\end{array}\right]$ is an even characteristic, so $a^{t} b=0\left(\in \mathbf{F}_{2}\right)$. These formulae are easily inverted to give

$$
\Theta[\sigma] \Theta[\sigma+a]=\frac{1}{2^{g}} \sum_{b}(-1)^{\sigma b} \theta\left[\begin{array}{l}
a \\
b
\end{array}\right]^{2} .
$$

It is easy to see that the $\theta[\Delta]^{2}$ span one-dimensional subrepresentations of $H_{g}$. In fact, using the classical formula one finds

$$
(s, x, u) \theta\left[\begin{array}{l}
a \\
b
\end{array}\right]^{2}=s^{2}(-1)^{u a+x b} \theta\left[\begin{array}{l}
a \\
b
\end{array}\right]^{2} .
$$

This implies that the $\theta\left[\begin{array}{l}a \\ b\end{array}\right]^{4}$ are Heisenberg invariants and thus are in $M_{2}\left(\Gamma_{g}(2)\right)$. More generally, we have

$$
\prod_{i}^{2 k} \theta\left[\left[_{b_{i}}^{a_{i}}\right]^{2} \in M_{2 k}\left(\Gamma_{g}(2)\right) \quad \text { iff } \quad \sum a_{i}=\sum b_{i}=0\left(\in \mathbf{F}_{2}\right) .\right.
$$

For example in case $g=1$ one has

$$
\theta\left[\begin{array}{l}
0 \\
0
\end{array}\right]^{2}=\Theta[0]^{2}+\Theta[1]^{2}, \quad \theta\left[\begin{array}{l}
0 \\
1
\end{array}\right]^{2}=\Theta[0]^{2}-\Theta[1]^{2}, \quad \theta\left[\begin{array}{l}
1 \\
0
\end{array}\right]^{2}=2 \Theta[0] \Theta[1],
$$


or, equivalently,

$$
\Theta[0]^{2}=\left(\theta\left[\begin{array}{l}
0 \\
0
\end{array}\right]^{2}+\theta\left[\begin{array}{l}
0 \\
1
\end{array}\right]^{2}\right) / 2, \quad \Theta[1]^{2}=\left(\theta\left[\begin{array}{l}
0 \\
0
\end{array}\right]^{2}-\theta\left[\begin{array}{l}
0 \\
1
\end{array}\right]^{2}\right) / 2, \quad \Theta[0] \Theta[1]=\theta\left[\begin{array}{l}
1 \\
0
\end{array}\right]^{2} / 2 .
$$

Note that upon substituting the first three relations in Jacobi's relation $\theta\left[\begin{array}{l}0 \\ 0\end{array}\right]^{4}=\theta\left[\begin{array}{l}0 \\ 1\end{array}\right]^{4}+\theta\left[\begin{array}{l}1 \\ 0\end{array}\right]^{4}$ one obtains a trivial identity in the $\Theta[\sigma]$ 's.

\subsection{The $\mathrm{O}^{+}$-invariants and $\mathrm{O}^{+}$-anti-invariants}

The function $\theta[0]^{4}=\left(\sum_{\sigma} \Theta[\sigma]^{2}\right)^{2}$ is Heisenberg invariant and thus defines a modular form of weight 2 on $\Gamma_{g}(2)$. For $g \in O^{+}(2 g)$ we have $g \cdot[0]=[0]$ and the explicit transformation formula for theta constants shows that $\theta[0]^{4}$ transforms by a non-trivial character which we denote by $\epsilon$ :

$$
\rho(g) \theta[0]^{4}=\epsilon(g) \theta[0]^{4}, \quad \epsilon: O^{+}(2 g) \longrightarrow\{ \pm 1\} .
$$

For $g \geq 3$, this homomorphism is the only non-trivial one-dimensional representation of $O^{+}(2 g)$ and its kernel is a simple group. In case $g=2$, Thomae's formula for $\theta[0]^{4}$ implies that $\epsilon$ is the product of the sign character on the subgroup $S_{3} \times S_{3}$ of $O^{+}(4)$ and $\epsilon(g)=1$ if $g=(14)(25)(36)$ where we identify $O^{+}(4)$ with a subgroup of $S_{6}$ as in Section 2.3 .

For applications to superstring measures, we will be particularly interested in the subspace of $O^{+}(2 g)$-anti-invariants in weight 6 :

$$
M_{6}\left(\Gamma_{g}(2)\right)^{\epsilon}:=\left\{f \in M_{6}\left(\Gamma_{g}(2)\right): \rho(g) f=\epsilon(g) f \quad \forall g \in O^{+}(2 g)\right\}
$$

and the space of $O^{+}$-invariants in weight 8 :

$$
M_{8}\left(\Gamma_{g}(2)\right)^{O^{+}}:=\left\{f \in M_{8}\left(\Gamma_{g}(2)\right): \rho(g) f=f \quad \forall g \in O^{+}(2 g)\right\} .
$$

It should be noted that $\operatorname{Sp}(2 g)$ permutes the $\theta[\Delta]^{4 k} \in M_{2 k}\left(\Gamma_{g}(2)\right)$, up to sign if $k$ is odd. Thus it is not hard to write down some invariants or anti-invariants, but the problem is to find all of them.

\subsection{The dimensions of the $\mathrm{O}^{+}-($anti)-invariants}

Once the decomposition of an $\operatorname{Sp}(2 g)$-representation into irreducibles is known, it is easy to find the dimension of the $O^{+}$-(anti)-invariants (although this is a case of overkill, since finding these $\mathrm{O}^{+}$-representations is much easier to do using the action of some generators of these groups). Recently 
Oura [21] used the methods from $[23,24]$ to determine the dimension of the $\mathrm{O}^{+}$-invariants in $M_{2 k}\left(\Gamma_{g}(2)\right)$ for small $k$ and $g$.

The dimension of the $\mathrm{O}^{+}$-invariants in $V$ is the multiplicity of the trivial representation 1 of $O^{+}$in the $O^{+}$-representation $\operatorname{Res}_{O^{+}}^{\mathrm{Sp}}(V)$ (the restriction of the representation from $\operatorname{Sp}(2 g)$ to $\left.O^{+}(2 g)\right)$ :

$$
\operatorname{dim} V^{O^{+}}=\left\langle\operatorname{Res}_{O^{+}}^{\mathrm{Sp}}(V), 1\right\rangle_{O^{+}}=\left\langle V, \operatorname{Ind}_{O^{+}}^{\mathrm{Sp}}(1)\right\rangle_{\mathrm{Sp}}
$$

where the second equality is Frobenius reciprocity. According to Frame [10] one has

$$
\operatorname{Ind}_{O^{+}}^{\mathrm{Sp}}(1)=\mathbf{1}+\sigma_{\theta}, \quad \operatorname{dim} \sigma_{\theta}=2^{g-1}\left(2^{g}+1\right)-1=\left(2^{g}-1\right)\left(2^{g}+2\right) / 2,
$$

where 1 is the trivial representation and $\sigma_{\theta}$ is an irreducible representation of $\operatorname{Sp}(2 g)$, note that $\operatorname{dim} \operatorname{Ind}_{O^{+}}^{\mathrm{Sp}}(1)=\left[\operatorname{Sp}(2 g): O^{+}(2 g)\right]=2^{g-1}\left(2^{g}+1\right)$. Thus if the multiplicity of $\mathbf{1}, \sigma_{\theta}$ in $V$ is $n_{1}, n_{\theta}$ respectively, then $\operatorname{dim} V^{O^{+}}=n_{1}+n_{\theta}$.

Similarly, the dimension of the $O^{+}$-anti-invariants in $V$ is the multiplicity of the representation $\epsilon$ of $O^{+}$in the $O^{+}$-representation $\operatorname{Res}_{O^{+}}^{\mathrm{Sp}}(V)$ :

$$
\operatorname{dim} V^{\epsilon}=\left\langle\operatorname{Res}_{O^{+}}^{\mathrm{Sp}}(V), \epsilon\right\rangle_{O^{+}}=\left\langle V, \operatorname{Ind}_{O^{+}}^{\mathrm{Sp}}(\epsilon)\right\rangle_{\mathrm{Sp}}
$$

According to Frame [10], the induced representation has two irreducible components:

$$
\operatorname{Ind}_{O^{+}}^{\mathrm{Sp}}(\epsilon)=\rho_{\theta} \oplus \rho_{r}, \quad\left\{\begin{array}{l}
\operatorname{dim} \rho_{\theta}=\left(2^{g}+1\right)\left(2^{g-1}+1\right) / 3 \\
\operatorname{dim} \rho_{r}=\left(2^{g}+1\right)\left(2^{g}-1\right) / 3
\end{array}\right.
$$

Thus if the multiplicity of $\rho_{\theta}, \rho_{r}$ in $V$ is $n_{\theta}, n_{r}$ respectively, then $\operatorname{dim} V^{\epsilon}=$ $n_{\theta}+n_{r}$.

\subsection{The $\operatorname{Sp}(2 g)$-representation on $M_{2}^{\theta}\left(\Gamma_{g}(2)\right)$}

The representation $\rho_{2}$ of $\operatorname{Sp}(2 g)$ on the subspace $M_{2}^{\theta}\left(\Gamma_{g}(2)\right) \subset M_{2}\left(\Gamma_{g}(2)\right)$ was shown to be irreducible and isomorphic to $\rho_{\theta}$ in [12].

We briefly recall the proof. As $\theta[0]^{4} \in M_{2}^{\theta}\left(\Gamma_{g}(2)\right)$ and $\theta[0]^{4}$ is an $O^{+}(2 g)$ anti-invariant, the representation $\epsilon$ of $\mathrm{O}^{+}$is a summand of the restriction of $\rho_{2}$ to $\mathrm{O}^{+}$:

$$
1 \geq\left\langle\operatorname{Res}_{O^{+}}^{\mathrm{Sp}}\left(\rho_{2}\right), \epsilon\right\rangle_{O^{+}}=\left\langle\rho_{2}, \operatorname{Ind}_{O^{+}}^{\mathrm{Sp}}(\epsilon)\right\rangle_{\mathrm{Sp}}
$$


As $\operatorname{Ind}_{O^{+}}^{\mathrm{Sp}}(\epsilon)=\rho_{\theta} \oplus \rho_{r}$, either $\rho_{\theta}$ or $\rho_{r}$ must be a summand of $\rho_{2}$. For $g \geq 3$, and using $\operatorname{dim} \mathbf{C}\left[\ldots, X_{\sigma}, \ldots\right]_{4}^{H_{g}}=\left(2^{g}+1\right)\left(2^{g-1}+1\right) / 3$ (cf. Section 2.8) we have

$$
\operatorname{dim} \rho_{r}>\operatorname{dim} \rho_{\theta}=\operatorname{dim} \mathbf{C}\left[\ldots, X_{\sigma}, \ldots\right]_{4}^{H_{g}} \geq \operatorname{dim} M_{2}^{\theta}\left(\Gamma_{g}(2)\right) .
$$

Therefore we have the irreducible representation $\rho_{2}=\rho_{\theta}$ on $M_{2}^{\theta}\left(\Gamma_{g}(2)\right)$ and $\mathbf{C}\left[\ldots, X_{\sigma}, \ldots\right]_{4}^{H_{g}}=M_{2}^{\theta}\left(\Gamma_{g}(2)\right.$ ) (so there are no $H_{g}$-invariant algebraic relations of degree 4$)$, moreover $\operatorname{dim} M_{2}^{\theta}\left(\Gamma_{g}(2)\right)=\left(2^{g}+1\right)\left(2^{g-1}+1\right) / 3$.

The induced representation $\operatorname{Ind}_{O^{+}}^{\mathrm{Sp}}(\epsilon)$ is realized on a vector space of dimension $\left[\operatorname{Sp}(2 g): O^{+}(2 g)\right]=2^{g-1}\left(2^{g}+1\right)$ which has a basis $e_{\Delta}$ parametrized by the even theta characteristics (or equivalently, by the cosets $\left.\operatorname{Sp}(2 g) / O^{+}(2 g)\right)$. There is an $\operatorname{Sp}(2 g)$-equivariant map

$$
\Phi: \operatorname{Ind}_{O^{+}}^{\mathrm{Sp}}(\epsilon) \longrightarrow M_{2}^{\theta}\left(\Gamma_{g}(2)\right), \quad e_{\Delta}=g \cdot e_{[0]} \longmapsto \rho_{2}(g) \theta[0]^{4}=\epsilon_{\Delta} \theta[\Delta]^{4},
$$

for some $\epsilon_{\Delta} \in\{ \pm 1\}$. The subrepresentation $\rho^{\theta}$ is mapped onto $M_{2}^{\theta}\left(\Gamma_{g}(2)\right)$ and the kernel of $\Phi$ is the subrepresentation $\rho_{r}$.

\subsection{The $\operatorname{Sp}(2 g)$-representation on $\operatorname{Sym}^{2}\left(M_{2}^{\theta}\left(\Gamma_{g}(2)\right)\right)$}

Frame has shown that the $\mathrm{Sp}(2 g)$-representation $\operatorname{Sym}^{2}\left(M_{2}^{\theta}\left(\Gamma_{g}(2)\right)\right)$ decomposes into irreducible representations as follows:

$$
\operatorname{Sym}^{2}\left(\rho_{\theta}\right)=\mathbf{1}+\sigma_{\theta}+\sigma_{c}, \quad \operatorname{dim} \sigma_{c}=2^{g-2}\left(2^{g}+1\right)\left(2^{g}-1\right)\left(2^{g}+2\right),
$$

and $\sigma_{\theta}$ as in Section 3.5. The functions $\theta[\Delta]^{8}$ are in $M_{4}^{\theta}\left(\Gamma_{g}(2)\right)$. They are permuted (without signs) by $\operatorname{Sp}(2 g)$ and span the subrepresentation $1+\sigma_{\theta}$. The trivial subrepresentation in $\operatorname{Sym}^{2}\left(M_{2}^{\theta}\left(\Gamma_{g}(2)\right)\right)$ is then spanned by the invariant $\sum_{\Delta} \theta[\Delta]^{8}$. It is easy to verify that the dimension of the image of $\operatorname{Sym}^{2}\left(M_{2}^{\theta}\left(\Gamma_{g}(2)\right)\right)$ is larger than $2^{g-1}\left(2^{g}+1\right)=\operatorname{dim}\left(\mathbf{1}+\sigma_{\theta}\right)$ for $g \geq 2$. As the multiplication map is $\operatorname{Sp}(2 g)$-equivariant it follows that $\operatorname{Sym}^{2}\left(M_{2}^{\theta}\left(\Gamma_{g}(2)\right)\right) \subset M_{4}^{\theta}\left(\Gamma_{g}(2)\right)$ (so if $f_{1}, \ldots, f_{N}$ is a basis of $M_{2}^{\theta}\left(\Gamma_{g}(2)\right)$ then the $f_{i} f_{j}$ are linearly independent).

\subsection{Decomposing representations of $\operatorname{Sp}(2 g)$}

Given a representation of a finite group on a complex vector space, one could determine the value of the character of the representation on each conjugacy 
class and then use the table of irreducible characters of the group to find the decomposition of the representation. However, it is very time consuming to compute these character values in our examples. Thus we take another approach, which has the additional advantage of finding explicitly certain subrepresentations.

There is one conjugacy class of $\operatorname{Sp}(2 g)$ which has only $2^{2 g}-1$ elements, the class of the transvections $t_{v}$ with $v \in \mathbf{F}_{2}^{2 g}-\{0\}$, see A.4. If $\rho: \operatorname{Sp}(2 g) \rightarrow$ $\mathrm{GL}(V)$ is a complex representation of $\mathrm{Sp}(2 g)$, the operator

$$
C=C_{\rho}:=\sum_{v \neq 0} \rho\left(t_{v}\right) \quad(\in \mathrm{GL}(V))
$$

obviously satisfies $\rho(g) C \rho(g)^{-1}=C$ for all $g \in \operatorname{Sp}(2 g)$. If $V=\oplus V_{i}^{n_{i}}$ is the decomposition of $V$ into irreducible representations $V_{i}, V_{i} \neq V_{j}$ if $i \neq j$, then, by Schur's lemma, $C$ must be scalar multiplication by a $\lambda_{i} \in \mathbf{C}$ on $V_{i}$. In particular, the eigenvalues of $C$ are the $\lambda_{i}$ with multiplicity $n_{i}\left(\operatorname{dim} V_{i}\right)$ (but it can happen that $\lambda_{i}=\lambda_{j}$ for $i \neq j$ ).

To find $\lambda_{i}$ we consider the trace of $C$ on $V_{i}$ : as the $t_{v}, v \neq 0$, are the elements of one conjugacy class,

$$
\operatorname{Tr}\left(C_{\mid V_{i}}\right)=\left(2^{2 g}-1\right) \operatorname{Tr}\left(\rho_{i}\left(t_{v}\right)\right)=\left(2^{2 g}-1\right) \chi_{i}\left(t_{v}\right),
$$

where $v$ is now one specific (but arbitrary) transvection and $\chi_{i}$ is the character of the irreducible representation $\rho_{i}$. On the other hand,

$$
\operatorname{Tr}\left(C_{\mid V_{i}}\right)=\left(\operatorname{dim} V_{i}\right) \lambda_{i}, \quad \text { hence } \quad \lambda_{i}=\frac{\left(2^{2 g}-1\right) \chi_{i}\left(t_{v}\right)}{\operatorname{dim} V_{i}} .
$$

Note that $\operatorname{ker}(C-\lambda I)$ will be the direct sum of the $V_{i}^{n_{i}}$ with $\lambda_{i}=\lambda$, so we do not only get information on the multiplicities of the irreducible constituents of $\rho$ but also on the corresponding subspaces of $V$.

\section{The case $g=1$}

\subsection{The geometry}

In case $g=1$, the holomorphic map

$$
\mathbf{H}_{1} \longrightarrow \mathbf{P}^{1}, \quad \tau \longmapsto(\Theta[0](\tau): \Theta[1](\tau))
$$


is non-constant and thus its image contains an open set of $\mathbf{P}^{1}$. This implies that there are no algebraic relations between the $\Theta[\sigma]$. In fact, the image is $\mathbf{P}^{1}-\{6$ points $\}$ (these points correspond to the zeroes of $\eta^{12}$, see below) and it is isomorphic to the modular curve $\mathbf{H}_{1} / \Gamma_{1}(2,4) \cong \mathbf{H}_{1} / \Gamma_{1}(4)$.

\subsection{The modular forms of even weight}

The dimension formula from Section 2.8 and the fact that $M_{2 k}^{\theta}\left(\Gamma_{1}(2)\right)=$ $M_{2 k}\left(\Gamma_{1}(2)\right)$ shows that $\operatorname{dim} M_{2 k}\left(\Gamma_{1}(2)\right)=k+1$. A basis of $M_{2}\left(\Gamma_{1}(2)\right)$ is given by the Heisenberg invariants $\Theta[0]^{4}+\Theta[1]^{4}$ and $(\Theta[0] \Theta[1])^{2}$. More generally, a basis of $M_{2 k}\left(\Gamma_{1}(2)\right)$ is given by the homogeneous polynomials of degree $k$ in these generators:

$$
\left(\Theta[0]^{4}+\Theta[1]^{4}\right)^{k}, \quad(\Theta[0] \Theta[1])^{2}\left(\Theta[0]^{4}+\Theta[1]^{4}\right)^{k-1}, \ldots,(\Theta[0] \Theta[1])^{2 k} .
$$

\subsection{The $\operatorname{Sp}(2)$ representation on $M_{2 k}\left(\Gamma_{1}(2)\right)$}

The functions $\theta[\Delta]^{4}$ are in $M_{2}\left(\Gamma_{1}(2)\right)$. The classical transformation theory of theta functions gives

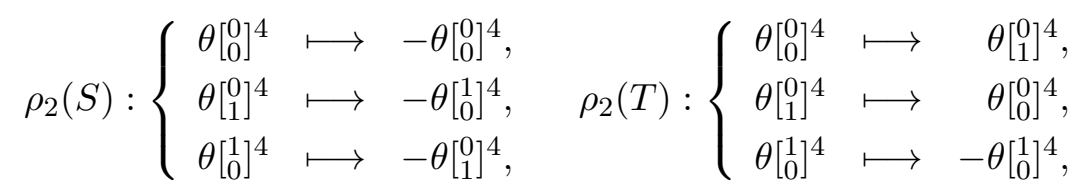

where $S, T$ are the standard generators of $\mathrm{SL}(2, \mathbf{Z})$. Recall the Jacobi relation $\theta\left[\begin{array}{l}0 \\ 0\end{array}\right]^{4}=\theta\left[\begin{array}{l}0 \\ 1\end{array}\right]^{4}+\theta\left[\begin{array}{l}1 \\ 0\end{array}\right]^{4}$ when computing the matrix of the $\rho_{2}(g)$ 's w.r.t. to the basis of $M_{2}\left(\Gamma_{1}(2)\right)$ given by $\theta\left[\begin{array}{l}0 \\ 0\end{array}\right]^{4}, \theta\left[\begin{array}{l}0 \\ 1\end{array}\right]^{4}$ for example.

In particular, $M_{2}\left(\Gamma_{1}(2)\right)$ is the unique irreducible two-dimensional representation $\rho_{[21]}$ of $S_{3}$, hence

$$
M_{2 k}\left(\Gamma_{1}(2)\right) \cong \operatorname{Sym}^{k}\left(\rho_{[21]}\right) .
$$

The group $O^{+}(2)$ is the group of order two generated by the image of $S \in$ $\mathrm{SL}(2, \mathbf{Z})$ in $\operatorname{Sp}(2)$.

\subsection{The decomposition of $M_{6}\left(\Gamma_{1}(2)\right)$}

We briefly discuss the decomposition of $M_{6}\left(\Gamma_{1}(2)\right)$ because it was (implicitly) used in our earlier papers. As $M_{6}\left(\Gamma_{1}(2)\right)=\operatorname{Sym}^{3}\left(M_{2}\left(\Gamma_{1}(2)\right)\right)$, it is the 
direct sum of the three irreducible representations of $S_{3}$ :

$$
M_{6}\left(\Gamma_{1}(2)\right) \cong \rho_{[3]} \oplus \rho_{[2,1]} \oplus \rho_{\left[1^{3}\right]},
$$

where $\rho_{\left[1^{3}\right]}$ is the sign representation of $S_{3}$ and $\rho_{[3]}$ is the trivial representation.

One verifies that

$$
\begin{gathered}
\rho_{[3]}=\left\langle\Theta[0]^{12}-33 \Theta[0]^{8} \Theta[1]^{4}-33 \Theta[0]^{4} \Theta[1]^{8}+\Theta[1]^{12}\right\rangle, \\
\rho_{\left[1^{3}\right]}=\left\langle\eta^{12}\right\rangle, \\
\rho_{[2,1]}=\left\{(a+b) \theta\left[\begin{array}{l}
0 \\
0
\end{array}\right]^{12}+a \theta\left[\begin{array}{l}
0 \\
1
\end{array}\right]^{12}+b \theta\left[\begin{array}{l}
1 \\
0
\end{array}\right]^{12}: a, b \in \mathbf{C}\right\},
\end{gathered}
$$

where we used a classical formula for the Dedekind $\eta$ function: $\eta^{3}=$ $\theta\left[\begin{array}{l}0 \\ 0\end{array}\right] \theta\left[\begin{array}{l}0 \\ 1\end{array}\right] \theta\left[\begin{array}{c}1 \\ 0\end{array}\right]$, so

$$
\begin{aligned}
\eta^{12} & \left.=\theta[0]_{0}^{0}\right]^{4} \theta\left[\left[_{1}^{0}\right]^{4} \theta\left[\begin{array}{l}
1 \\
0
\end{array}\right]^{4}\right. \\
& =\left(\Theta[0]^{2}+\Theta[1]^{2}\right)^{2}\left(\Theta[0]^{2}-\Theta[1]^{2}\right)^{2}(2 \Theta[0] \Theta[1])^{2} .
\end{aligned}
$$

The function $\eta^{12}$ is a modular form of weight 6 on $\Gamma_{1}(2)$; viewed as homogeneous polynomial of degree 6 , it vanishes in the six points $(1: 0),(0: 1)$, $\left(1: i^{k}\right)$, with $k=0,1,2,3$ and $i^{2}=-1$, in $\mathbf{P}^{1}$.

The subspace of $O^{+}(2)$-anti-invariants is:

$$
M_{6}\left(\Gamma_{g}(2)\right)^{\epsilon}=\left\langle\eta^{12}, f_{21}:=2 \theta\left[\begin{array}{l}
0 \\
0
\end{array}\right]^{12}+\theta\left[\begin{array}{l}
0 \\
1
\end{array}\right]^{12}+\theta\left[\begin{array}{l}
1 \\
0
\end{array}\right]^{12}\right\rangle,
$$

the function $f_{21}$ lies in the two-dimensional irreducible subrepresentation.

\section{The case $g=2$}

\subsection{The geometry}

In case $g=2$, the four $\Theta[\sigma]$ 's define a holomorphic map

$$
\mathbf{H}_{2} \longrightarrow \mathbf{P}^{3}, \quad \tau \longmapsto(\Theta[00](\tau): \Theta[01](\tau): \Theta[10](\tau): \Theta[11](\tau))
$$

which factors over $\mathbf{H}_{2} / \Gamma_{2}(2,4)$. Its image contains an open subset of $\mathbf{P}^{3}$, hence there are no algebraic relations between the $\Theta[\sigma]$ 's. Results of Igusa imply that this map induces an isomorphism between the Satake compactification of $\mathbf{H}_{2} / \Gamma_{2}(2,4)$ and $\mathbf{P}^{3}$. In particular, the image of the map 
is $\mathbf{H}_{2} / \Gamma_{2}(2,4)$ and it is $\mathbf{P}^{3}$ minus the union of 15 pairs of lines. The pairs of lines are the pairs of eigenspaces of the 15 elements $(1, x, u)$, with $(x, u) \in \mathbf{F}_{2}^{4}-\{0\}$, in the Heisenberg group acting on $\mathbf{P}^{3}$. For example $(1,(0,0),(1,0))$ maps $\Theta[a b]$ to $(-1)^{a} \Theta[a b]$ and thus has two eigenlines parametrized by $(s: t: 0: 0)$ and $(0: 0: s: t)$ where $(s: t) \in \mathbf{P}^{1}$.

The quotient of $\mathbf{P}^{3}$ by the Heisenberg group is the Satake compactification of $\mathbf{H}_{2} / \Gamma_{2}(2)$. The ring of Heisenberg invariants can be shown to be generated by five polynomials $p_{0}, \ldots, p_{4}$, homogeneous of degree 4 , in the $\Theta[\sigma]$ 's:

$$
\mathbf{C}[\ldots, \Theta[\sigma], \ldots]^{H_{2}}=\mathbf{C}\left[p_{0}, \ldots, p_{4}\right]
$$

where the $p_{i}$ are defined as

$$
\begin{aligned}
& p_{0}=\Theta[00]^{4}+\Theta[01]^{4}+\Theta[10]^{4}+\Theta[11]^{4}, \\
& p_{1}=2\left(\Theta[00]^{2} \Theta[01]^{2}+\Theta[10]^{2} \Theta[11]^{2}\right), \\
& p_{2}=2\left(\Theta[00]^{2} \Theta[10]^{2}+\Theta[01]^{2} \Theta[11]^{2}\right), \\
& p_{3}=2\left(\Theta[00]^{2} \Theta[11]^{2}+\Theta[01]^{2} \Theta[10]^{2}\right), \\
& p_{4}=4 \Theta[00] \Theta[01] \Theta[10] \Theta[11] .
\end{aligned}
$$

Thus the quotient map is given by these quartics $\mathbf{P}^{3} \rightarrow \mathbf{P}^{4}$. Equivalently, $\mathbf{H}_{2} / \Gamma_{2}(2)$ is the image of the holomorphic map given by the quartics in the $\Theta[\sigma](\tau)$ 's above. The 15 pairs of eigenlines map to 15 lines in $\mathbf{P}^{4}$, for example both $(s: t: 0: 0)$ and $(0: 0: s: t)$ map to $\left(s^{4}+t^{4}: 2 s^{2} t^{2}: 0: 0: 0\right)$ which is the general point on the line parametrized by $(u: v: 0: 0: 0)$ with $(u: v) \in \mathbf{P}^{1}$. The image of $\mathbf{P}^{3}$ is defined by a quartic polynomial $f_{4}$ in five variables, the Igusa quartic (cf. [3]), that is $f_{4}\left(p_{0}, \ldots, p_{4}\right)$ is identically zero. It follows that, as a graded ring,

$$
\underset{k=0}{\oplus} M_{2 k}\left(\Gamma_{2}(2)\right) \cong \mathbf{C}\left[y_{0}, \ldots, y_{4}\right] /\left(f_{4}\left(y_{0}, \ldots, y_{4}\right)\right) .
$$

In particular, $\quad \operatorname{dim} M_{2}=5, \quad \operatorname{dim} M_{4}=15, \quad \operatorname{dim} M_{6}=35, \quad \operatorname{dim} M_{8}=$ $70-1=69$.

The singular locus of the image of $\mathbf{P}^{3}$, i.e., of the threefold in $\mathbf{P}^{4}$ defined by $f_{4}=0$, is the union of the 15 "boundary" lines. In [3] it was shown that the five partial derivatives $\partial f_{4} / \partial y_{i}\left(p_{0}, \ldots, p_{4}\right)$, span a five dimensional space of modular forms of weight 6 on $\Gamma_{2}(2)$ which is precisely the space spanned by the $\Xi_{6}[\delta]$ 's, the modular forms introduced by D'Hoker and Phong in [6]. Hence the $\Xi_{6}[\delta]$ are zero on the boundary, that is, they are cusp forms. 


\subsection{The $\mathrm{Sp}(4)$-representations on the $M_{2 k}\left(\Gamma_{2}(2)\right)$}

Using the isomorphism $\operatorname{Sp}(4) \cong S_{6}$, the irreducible representations of $\operatorname{Sp}(4)$ are labelled by partitions of 6 . One has the identifications (cf. [3], one finds $\rho_{\theta}$ from the representation on $V_{\theta}:=M_{2}\left(\Gamma_{2}(2)\right), \rho_{\theta}+\rho_{r}$ is the representation on the $\theta[\delta]^{12}$, which is $\operatorname{Ind}_{O^{+}}^{\mathrm{Sp}}(\epsilon)$ and $1+\sigma_{\theta}$ is the permutation representation on the 10 even $\theta[\delta]^{8}$, hence its trace must be $\geq 0$ on each conjugacy class):

$$
\rho_{\theta}=\rho_{\left[2^{3}\right]}, \quad \rho_{r}=\rho_{\left[21^{4}\right]}, \quad \sigma_{\theta}=\rho_{[42]} .
$$

Using the representation theory of $S_{6}$ one finds:

$$
\begin{aligned}
& M_{2}\left(\Gamma_{2}(2)\right) \cong \rho_{\left[2^{3}\right]}, \\
& M_{4}\left(\Gamma_{2}(2)\right) \cong \operatorname{Sym}^{2}\left(\rho_{\left[2^{3}\right]}\right)=\mathbf{1}+\rho_{[42]}+\rho_{\left[2^{3}\right]}, \\
& M_{6}\left(\Gamma_{2}(2)\right) \cong \operatorname{Sym}^{3}\left(\rho_{\left[2^{3}\right]}\right)=\mathbf{1}+2 \rho_{\left[2^{3}\right]}+\rho_{\left[2^{4}\right]}+\rho_{[42]}+\rho_{\left[31^{3}\right]}, \\
& M_{8}\left(\Gamma_{2}(2)\right) \cong \operatorname{Sym}^{4}\left(\rho_{\left[2^{3}\right]}\right)-\mathbf{1}=\mathbf{1}+3 \rho_{\left[2^{3}\right]}+3 \rho_{[42]}+\rho_{\left[31^{3}\right]}+\rho_{[321]} .
\end{aligned}
$$

Explicit functions in the various subrepresentations of $M_{6}\left(\Gamma_{2}(2)\right)$ are given in [3].

\section{The case $g=3$}

\subsection{The geometry}

In case $g=3$, the $8 \Theta[\sigma]$ 's define a holomorphic map

$$
\mathbf{H}_{3} \longrightarrow \mathbf{P}^{7}, \quad \tau \longmapsto(\Theta[000](\tau): \ldots: \Theta[111](\tau))
$$

which factors over $\mathbf{H}_{3} / \Gamma_{3}(2,4)$. The image of this map is a 6 -dimensional quasi-projective variety $Z^{0}$ whose closure $Z$ is defined by a homogeneous (Heisenberg invariant) polynomial $F_{16}$ of degree 16 , see Section 6.10. In particular, the holomorphic function $\tau \mapsto F_{16}(\ldots, \Theta[\sigma](\tau), \ldots)$ is identically zero on $\mathbf{H}_{3}$. The complement of $Z^{0}$ in $Z$ is the union of 63 pairs of $\mathbf{P}^{3}$ 's, which are the eigenspaces of elements in the Heisenberg group.

\subsection{Modular forms}

As there is only one relation of degree 16 and the quotient ring is normal we get $[23,24]$ :

$$
M_{2 k}\left(\Gamma_{3}(2)\right)=M_{2 k}^{\theta}\left(\Gamma_{3}(2)\right)=\left(\mathbf{C}[\ldots, \Theta[\sigma], \ldots]_{4 k}\right)^{H_{3}}
$$


with

$$
\begin{aligned}
\left(\mathbf{C}[\ldots, \Theta[\sigma], \ldots]_{4 k}\right)^{H_{3}} & \\
\quad & = \begin{cases}\left(\mathbf{C}\left[\ldots, X_{\sigma}, \ldots\right]_{4 k}\right)^{H_{3}}, & k \leq 3, \\
\left(\mathbf{C}\left[\ldots, X_{\sigma}, \ldots\right]_{4 k}\right)^{H_{3}} / F_{16}\left(\mathbf{C}\left[\ldots, X_{\sigma}, \ldots\right]_{4 k-16}\right)^{H_{3}}, & k \geq 4 .\end{cases}
\end{aligned}
$$

\subsection{Weight 2}

From Section 3.6 we know that $M_{2}\left(\left(\Gamma_{3}(2)\right)\right.$, a 15 -dimensional vector space, is an irreducible $\operatorname{Sp}(6)$-representation, denoted by $\rho_{\theta}$. As $\operatorname{Sp}(6)$ has a unique irreducible representation of dimension 15 , denoted by $\mathbf{1 5}_{a}$ in [9], it follows that $\rho_{\theta} \cong \mathbf{1 5}_{a}$.

\subsection{Weight 4}

From Section 3.7 we know that $\operatorname{Sym}^{2}\left(M_{2}\left(\Gamma_{3}(2)\right)\right) \subset M_{4}\left(\Gamma_{3}(2)\right)$ and as an $\mathrm{Sp}(6)$-representation we have:

$$
\operatorname{Sym}^{2}\left(M_{2}\left(\Gamma_{3}(2)\right)\right):=\operatorname{Sym}^{2}\left(\mathbf{1 5}_{a}\right)=\mathbf{1}+\mathbf{3 5}_{b}+\mathbf{8 4}_{a} .
$$

The invariant subspace is spanned by $\sum_{\Delta} \theta[\Delta]^{8}$ and the subrepresentation $\mathbf{1}+\mathbf{3 5}_{b}$ is spanned by the $36 \theta[\Delta]^{8}$ 's which are permuted by $\operatorname{Sp}(6)$.

We recall the following relation, which holds for all $\tau \in \mathbf{H}_{3}$ :

$$
\begin{aligned}
& r_{1}-r_{2}=r_{3}, \quad \text { with } \\
& r_{1}=\prod_{a, b \in \mathbf{F}_{2}} \theta\left[\begin{array}{l}
000 \\
0 a b
\end{array}\right](\tau), \quad r_{2}=\prod_{a, b \in \mathbf{F}_{2}} \theta\left[\begin{array}{l}
000 \\
1 a b
\end{array}\right](\tau), \quad r_{3}=\prod_{a, b \in \mathbf{F}_{2}} \theta\left[\begin{array}{l}
100 \\
0 a b
\end{array}\right](\tau) .
\end{aligned}
$$

From this we deduce that $2 r_{1} r_{2}=r_{1}^{2}+r_{2}^{2}-r_{3}^{2}$. Thus $r_{1} r_{2}$, a product of 8 distinct $\theta[\Delta]$ 's, is a linear combination of three products of four theta squares. The sum of the four characteristics in each product is zero, hence

$$
r_{1} r_{2}=\prod_{a, b, c \in \mathbf{F}_{2}} \theta\left[\begin{array}{l}
000 \\
a b c
\end{array}\right] \in M_{4}\left(\Gamma_{3}(2)\right) .
$$

We verified that under the action of $\operatorname{Sp}(6)$ on $r_{1} r_{2}$ one obtains 135 functions which are a basis of $M_{4}\left(\Gamma_{3}(2)\right)$ and which are permuted (without signs) by $\operatorname{Sp}(6)$. 
Let $P \subset \mathrm{Sp}(6)$ be the stabilizer of $r_{1} r_{2}$, it consists of the matrices with blocks $A, \ldots, D$ with $C=0$. There are no non-trivial homomorphisms $P \rightarrow$ $G L_{1}(\mathbf{C})=\mathbf{C}^{*}$, because these factor over $\mathrm{SL}\left(3, \mathbf{F}_{2}\right)$ (with $P$ as before, one maps the matrix first to $A$ ) and this is a simple group (of order 168). Thus any $g \in P$ acts as the identity on $r_{1} r_{2}$. By Frobenius reciprocity one can then identify the representation of $\operatorname{Sp}(6)$ on $M_{4}\left(\Gamma_{3}(2)\right)$ with $\operatorname{Ind}_{P}^{\mathrm{Sp}}\left(1_{P}\right)$, this representation is (cf. [9, p. 113])

$$
\begin{aligned}
M_{4}\left(\Gamma_{3}(2)\right) & \cong \operatorname{Ind}_{P}^{\mathrm{Sp}}\left(1_{P}\right) \cong \mathbf{1}+\mathbf{3 5}_{b}+\mathbf{8} \mathbf{4}_{a}+\mathbf{1 5}_{a} \\
& \cong \operatorname{Sym}^{2}\left(M_{2}\left(\Gamma_{3}(2)\right)\right)+\mathbf{1 5}_{a} .
\end{aligned}
$$

In particular, there is a unique complementary 15-dimensional subspace which is $\mathrm{Sp}(6)$-invariant; the representation on this subspace must be $\mathbf{1 5}_{a} \cong \rho_{\theta}$. An isomorphism of representations, which uses some geometry of quadrics, can be obtained as follows.

Let $L \subset \mathbf{F}_{2}^{6}$ be a Lagrangian subspace (so $L \cong \mathbf{F}_{2}^{3}$ and $E(v, w)=0$ for all $v, w \in L$ ). Then there are eight even quadrics $Q$ such that $L \subset Q$ (cf. [4, Appendix A]). In case $L=L_{0}=\left\{\left(v^{\prime}, v^{\prime \prime}\right): v^{\prime \prime}=0\right\}$, the 8 even quadrics containing $L_{0}$ have the same characteristics as the eight theta constants in $r_{1} r_{2}$. Each of the 135 functions in the $\mathrm{Sp}(6)$-orbit of $r_{1} r_{2}$ can thus be written as

$$
P_{L}= \pm \prod_{Q \supset L} \theta\left[\Delta_{Q}\right]
$$

for a unique Lagrangian subspace $L$, where the product is over the eight quadrics containing $L$ and the sign is determined by the condition that it is +1 if $L=L_{0}$ and that $P_{L}=\rho(g) P_{L_{0}}$ for some $g \in \operatorname{Sp}(6)$.

Using this description of the basis of $M_{4}\left(\Gamma_{3}(2)\right)$ it is not hard to write down the (unique) $O^{+}$-anti-invariant. Recall that $O^{+}$is the stabilizer of the even characteristic [0] and let $Q_{0}$ be the corresponding even quadric. An even (=split) quadric $Q$ in $\mathbf{F}_{2}^{6}$ (cf. Appendix A.1) contains 30 Lagrangian subspaces, 15 in each ruling $\left(L, L^{\prime} \subset Q\right.$ are in the same ruling if $L \cap L^{\prime}$ is one-dimensional). Let $P[0]$ be the sum of the $15 P_{L}$ 's from one ruling minus the sum of the $15 P_{L}$ 's from the other ruling of $Q_{0}$. Then $P[0]$ transforms with the representation $\epsilon$ of $O^{+}$. Thus the subrepresentation of $M_{4}\left(\Gamma_{3}(2)\right)$ generated by $P[0]$ is contained in $\operatorname{Ind}_{O^{+}}^{\mathrm{Sp}}(\epsilon)$ and thus it must be $\rho_{\theta}=\mathbf{1 5}_{a}, \rho_{r}=\mathbf{2 1}_{b}$ or their direct sum. As only $\mathbf{1 5}_{a}$ is a component of the representation on $M_{4}\left(\Gamma_{3}(2)\right)$, we conclude that the subrepresentation generated by $P[0]$ is isomorphic to $\mathbf{1 5}_{a}$ and thus is complementary to $\operatorname{Sym}^{2}\left(M_{2}\left(\Gamma_{3}(2)\right)\right)$. 


\subsection{The $\operatorname{Sp}(6)$-representation on $M_{6}\left(\Gamma_{3}(2)\right)$}

We verified that the 680 products $P_{i} P_{j} P_{k}(0 \leq i \leq j \leq k \leq 14)$, where the $P_{i}$ are basis of $M_{2}\left(\Gamma_{3}(2)\right)$, are linearly independent in the 870 -dimensional vector space $\left(\mathbf{C}[\ldots, \Theta[\sigma], \ldots]_{8}\right)^{H_{g}}$. We denote the subspace which they span by

$$
\operatorname{Sym}^{3}\left(M_{2}\left(\Gamma_{3}(2)\right)\right):=\left\langle P_{i} P_{j} P_{k}: P_{i}, P_{j}, P_{k} \in M_{2}\left(\Gamma_{3}(2)\right)\right\rangle .
$$

As $\operatorname{Sp}\left(6, \mathbf{F}_{2}\right)$-representation we have $M_{2}\left(\Gamma_{3}(2)\right) \cong \mathbf{1 5}_{a}$, and this implies

$$
\operatorname{Sym}^{3}\left(M_{2}\left(\Gamma_{3}(2)\right)\right) \cong 2 \cdot \mathbf{1 5}_{a}+\mathbf{2 1}_{b}+\mathbf{3 5}_{b}+\mathbf{8 4 _ { a }}+\mathbf{1 0 5}_{c}+\mathbf{1 8 9}_{c}+\mathbf{2 1 6} \mathbf{6}_{a}
$$

To decompose all of $V=M_{6}\left(\Gamma_{3}(2)\right)$ we use operator $C=C_{V}$ as in 2.8. A computer computation showed that the eigenvalues $\lambda$ with multiplicities $m_{\lambda}$ of $C$ on $V$ are

$$
\begin{aligned}
\left(\lambda, m_{\lambda}\right): & (63,1), \quad(27,35), \quad(3,378), \quad(-7,216), \quad(-13,189), \\
& (-21,30), \quad(-33,21) .
\end{aligned}
$$

Obviously $(63,1)$ corresponds to the one-dimensional trivial representation. Using the character table of $\mathrm{Sp}(6)$ in [9], p. 114-115 (where $t_{v}$ is in the 16th conjugacy class labelled $1^{-5} 2^{6}$ ), one finds unique irreducible representations $\rho$ of dimension $d_{\lambda}$ such that $C_{\rho}$ has eigenvalue $\lambda$ in the cases $\left(\lambda, d_{\lambda}\right)=(-7,216),(-13,189),(-33,21)$. It follows that the irreducible representations $\mathbf{2 1 6}, \mathbf{1 8 9}_{c}$ and $\mathbf{2 1}_{b}$ occur in $M_{6}\left(\Gamma_{3}(2)\right)$, all three with multiplicity one. The irreducible representations $\rho$ for which $C_{\rho}$ has eigenvalue $\lambda=27$ are $\mathbf{2 1}_{a}$ and $\mathbf{3 5}_{b}$. As $C$ has a 35-dimensional eigenspace with $\lambda=27$, the representation $\mathbf{3 5}_{b}$ occurs with multiplicity one in $M_{6}\left(\Gamma_{3}(2)\right)$. The irreducible representations $\rho$ for which $C_{\rho}$ has eigenvalue $\lambda=3$ are: $\mathbf{1 0 5}_{c}, \mathbf{8 4}_{a}$, $\mathbf{4 2 0}_{a}, \mathbf{2 1 0}_{b}$. This gives two possibilities for the decomposition of the 378dimensional eigenspace with $\lambda=3: 2 \cdot \mathbf{1 0 5}_{c}+2 \cdot \mathbf{8 4}_{a}$ or $\mathbf{2 1 0}_{b}+2 \cdot \mathbf{8 4}_{a}$. As we saw above, $\mathbf{1 0 5}_{c}$ is an irreducible component of $\operatorname{Sym}^{3}\left(\mathbf{1 5}_{a}\right) \subset M_{6}\left(\Gamma_{3}(2)\right)$, hence we conclude that $2 \cdot \mathbf{1 0 5}_{c}+2 \cdot \mathbf{8 4}_{a}$ is a summand of $M_{6}\left(\Gamma_{3}(2)\right)$. The irreducible representations $\rho$ for which $C_{\rho}$ has eigenvalue $\lambda=-21$ are: $\mathbf{1 0 5}_{a}$ and $\mathbf{1 5}_{a}$. As $C$ has a 30-dimensional eigenspace with $\lambda=-21$ it follows that $2 \cdot \mathbf{1 5}_{a}$ is the representation on this eigenspace.

Combining these results with the decomposition of $\operatorname{Sym}^{3}\left(\mathbf{1 5}_{a}\right)$ above, we get

$$
M_{6}\left(\Gamma_{3}(2)\right)=\operatorname{Sym}^{3}\left(\mathbf{1 5}_{a}\right)+\mathbf{1}+\mathbf{8 4}_{a}+\mathbf{1 0 5}_{c} .
$$




\subsection{The invariant in $M_{6}\left(\Gamma_{3}(2)\right)$}

The trivial subrepresentation corresponds to an $\mathrm{Sp}(6)$-invariant modular form, that is, to a modular form of weight 6 for $\Gamma_{3}$ whose existence follows already from the dimension formula for $M_{k}\left(\Gamma_{3}\right)$ found by Tsuyumine [27], cf. $[24$, p. 188].

Our results on the decomposition of $M_{2 k}\left(\Gamma_{3}(2)\right)$ for $k=1,2$ suggest a way to write down this invariant $G$ which may be of some interest. Both $M_{2}\left(\Gamma_{3}(2)\right)$ and $M_{4}\left(\Gamma_{3}(2)\right)$ have a unique copy of the the $\operatorname{Sp}(6)$-representation $\rho_{\theta}=15_{a}$. The representation $M_{2}\left(\Gamma_{3}(2)\right) \cong \rho_{\theta}$ contains the 36 functions $\theta[\Delta]^{4}$ and in 6.4 we identified a function $P[0]$ in $\mathbf{1 5}_{a} \subset M_{4}\left(\Gamma_{3}(2)\right)$ on which $O^{+}(6)$ acts via the character $\epsilon$, as it does on $\theta[0]^{4}$. Since the subspace of $O^{+}$-anti-invariants in $\rho_{\theta}$ is one dimensional, we get an isomorphism of $\operatorname{Sp}(6)$-representations $\Phi$ by defining

$$
\Phi: M_{2}\left(\Gamma_{3}(2)\right) \stackrel{\cong}{\longrightarrow} \mathbf{1 5}_{a}\left(\subset M_{4}\left(\Gamma_{3}(2)\right)\right) \quad \Phi\left(\rho_{2}(g) \theta[0]^{4}\right):=\rho_{4}(g) P[0] .
$$

Now let $P[\Delta]:=\Phi\left(\theta[\Delta]^{4}\right)$. Then the function $G:=\sum_{\Delta} \theta[\Delta]^{4} P[\Delta] \in$ $M_{6}\left(\Gamma_{3}(2)\right)$ corresponds to the function $\sum_{\Delta} \theta[\Delta]^{8} \in \operatorname{Sym}^{2}\left(\mathbf{1 5}_{a}\right)$, which is an $\mathrm{Sp}(6)$-invariant. Thus $G$ is an $\mathrm{Sp}(6)$-invariant and an explicit verification showed that $G \neq 0$.

\subsection{Asyzygous sextets}

The subrepresentation space $\mathbf{1 0 5}_{c}$ in the complement of $\operatorname{Sym}^{3}\left(\mathbf{1 5}_{a}\right)$ is quite interesting and, as we will show, has in fact been studied by D'Hoker and Phong in [8]. Following [8] we consider sets $S=\left\{\Delta_{1}, \ldots, \Delta_{6}\right\}$ of six totally asyzygous (even) characteristics, that is, $\Delta_{i}+\Delta_{j}+\Delta_{k}$ is odd for distinct $i, j, k$. An example of such a sextet of even characteristics is

$$
\left.S_{0}:=\left\{\begin{array}{l}
110 \\
110
\end{array}\right], \quad\left[\begin{array}{l}
110 \\
111
\end{array}\right], \quad\left[\begin{array}{l}
111 \\
110
\end{array}\right], \quad\left[\begin{array}{l}
101 \\
101
\end{array}\right], \quad\left[\begin{array}{l}
101 \\
111
\end{array}\right], \quad\left[\begin{array}{l}
111 \\
101
\end{array}\right]\right\}
$$

Note that the six characteristics in the sextet $S_{0}$ are of the form $\left[\begin{array}{l}1 a b \\ 1 c d\end{array}\right]$ where $\left[\begin{array}{c}a b \\ c d\end{array}\right]$ runs over the six odd theta characteristics in genus 2. It is well known that the sum of any three odd characteristics in genus two is even, so the sum of any three of these six characteristics for $g=3$ is indeed odd. There are 336 asyzygous sextets and $\mathrm{Sp}(6)$ acts transitively on the 336 totally asyzygous sextets. The sum of the six characteristics in such a sextet is zero (in $\mathbf{F}_{2}$ ), hence to a sextet $S$ we can associate a modular form $F_{S}$ of weight 6 
on $\Gamma_{3}(2)$ :

$$
F_{S}:=\prod_{\Delta \in S} \theta[\Delta]^{2} \quad\left(\in M_{6}\left(\Gamma_{3}(2)\right)\right) .
$$

Using the classical theta formula 3.3, each $F_{S}$ corresponds to a Heisenberg invariant homogeneous polynomial of degree 12 in the $\Theta[\sigma]$ 's. A computer computation shows that the 336 functions $F_{S}$ span a 105 -dimensional vector space, which we denote by

$$
W_{\mathrm{as}}:=\left\langle F_{S}: S \text { totally asyzygous sextet }\right\rangle \quad\left(\subset M_{6}\left(\Gamma_{3}(2)\right)\right) .
$$

We verified that $\operatorname{Sym}^{3}\left(M_{2}\left(\Gamma_{3}(2)\right)\right) \cap W_{\text {as }}=\{0\}$ and that $C$ is multiplication by 3 on $W_{\text {as }}$, hence $W_{\text {as }} \cong \mathbf{1 0 5}_{c}$. Therefore $W_{\text {as }}$ is the 105-dimensional irreducible representation in the complement of $\operatorname{Sym}^{3}\left(\mathbf{1 5}_{a}\right)$.

One of the results in [8] is that there are no functions in $W_{\text {as }}$ which transform as $\theta[0]^{4}$ under $O^{+}(6)$. This is now clear, since such a function would generate a subrepresentation of $\operatorname{Ind}_{O^{+}}^{\mathrm{Sp}}(\epsilon)=\mathbf{1 5}_{a} \oplus \mathbf{2 1}_{b}$, which contradicts the fact that $W_{\mathrm{as}} \cong \mathbf{1 0 5}_{c}$.

\subsection{Cusp forms in $W_{\text {as }}$}

A modular form $f \in M_{2 k}\left(\Gamma_{g}(2)\right)$ is called a cusp form if, for all $M \in \Gamma_{g}(1)$, and all $\tau_{g-1} \in \mathbf{H}_{g-1}$ one has, with $\tau_{1} \in \mathbf{H}_{1}$ :

$$
\lim _{\tau_{1} \rightarrow i \infty} f\left(M \cdot \tau_{g-1,1}\right)=0, \quad \text { with } \quad \tau_{g-1,1}=\left(\begin{array}{cc}
\tau_{g-1} & 0 \\
0 & \tau_{1}
\end{array}\right) .
$$

We now show that the $F_{S}$ are cusp forms. The group $\operatorname{Sp}(2)$ acts transitively on these functions, so it suffices to show that $F_{S_{0}}$ is a cusp form. But $F_{S_{0}}\left(M \cdot \tau_{g-1,1}\right)= \pm F_{T}\left(\tau_{g-1,1}\right)$ where $T$ is sextet $M^{-1} \cdot \Delta_{i}$, where the $\Delta_{i}, 1 \leq i \leq 6$ are the characteristics in $S_{0}$. So we must show that $\lim _{\tau_{1} \rightarrow \mathrm{i} \infty} F_{S}\left(\tau_{g-1,1}\right)=0$ for all 336 asyzygous sextets $S$. As the limit $\theta\left[\begin{array}{l}a b c \\ \text { def }\end{array}\right]\left(\tau_{g-1,1}\right)=0$ for all $\tau_{g-1}$ iff $c=1$, we must verify that each sextet has at least one characteristic with $c=1$. This can be done by computer, and we did that (there is a more elegant method, using that the asyzygous sextets correspond to decompositions $\mathbf{F}_{2}^{6}=V_{1} \oplus V_{2}$ with $V_{i}$ symplectic subspaces of dimension $V_{i}$, the condition that $\theta[\Delta] \rightarrow 0$ is just that $v=\left(\begin{array}{c}001 \\ 000\end{array}\right) \in \mathbf{F}_{2}^{6}$ does not lie in $Q_{\Delta}$, etc.). 


\subsection{Miyawaki's $F_{12}$}

The cusp forms $F_{S}$ are permuted, up to signs, by the elements of $\operatorname{Sp}(6)$, thus the sum (over the 336 asyzygous sextets) of their squares

$$
F_{12}=\sum_{S} F_{S}^{2}
$$

is an $\mathrm{Sp}(6)$-invariant cusp form of weight 12 , if it is not identically zero on $\mathbf{H}_{3}$. To exclude that possibility we computed the degree 24 polynomial in the $X_{\sigma}$ corresponding to $F_{12}$ and verified that the result is not a multiple of the polynomial $F_{16}$ (we actually put $X_{\sigma}=1$ except for $X_{111}$ in this computation). Thus $F_{12}$ is a cusp form of weight 12 on $\Gamma_{3}(1)$ and was first discovered by Miyawaki [20]. Recently Heim [15] determined the spinor L-function of this cusp form which is of interest for arithmetical applications.

\subsection{The $\operatorname{Sp}(6)$-representation on $M_{8}\left(\Gamma_{3}(2)\right)$}

The modular forms of weight 8 on $\Gamma_{3}(2)$ can be described as follows:

$$
\mathbf{C}\left[\ldots, X_{\sigma}, \ldots\right]_{16}^{H_{g}} \cong M_{8}\left(\Gamma_{3}(2)\right) \oplus\left\langle F_{16}\right\rangle,
$$

where $F_{16}$ is a homogeneous polynomial of degree 16 in the $X_{\sigma}$ such that $F_{16}(\ldots, \Theta[\sigma](\tau), \ldots)=0$ for all $\tau \in \mathbf{H}_{3}$, see $[13,14$, Section 5.1]. This polynomial may be written as

$$
F_{16}=8 \sum_{\Delta} \theta[\Delta]^{16}-\left(\sum_{\Delta} \theta[\Delta]^{8}\right)^{2}, \quad \theta\left[\left[_{\epsilon^{\prime}}^{\epsilon}\right]^{2}=\sum_{\sigma}(-1)^{\sigma \epsilon^{\prime}} X_{\sigma} X_{\sigma+\epsilon}\right.
$$

so one substitutes $X_{\sigma}$ rather than $\Theta[\sigma]$ in the classical theta formula. In particular,

$$
\operatorname{dim} M_{8}\left(\Gamma_{3}(2)\right)=3993-1=3992 .
$$

We computed the dimension of the image, denoted by $\operatorname{Sym}^{4}\left(M_{2}\left(\Gamma_{3}(2)\right)\right)_{0}$, of $\operatorname{Sym}^{4}\left(M_{2}\left(\Gamma_{3}(2)\right)\right)$ in this 3992-dimensional vector space (that is, of the space spanned by the products $P_{i} P_{j} P_{k} P_{l}$, for a basis $P_{i} \in \mathbf{C}\left[\ldots, X_{\sigma}, \ldots\right]_{4}^{H_{g}}$, $1 \leq i \leq 15$, the image includes $F_{16}$ ). There are 27 independent relations, thus

$$
\operatorname{dim} \operatorname{Sym}^{4}\left(M_{2}\left(\Gamma_{3}(2)\right)\right)_{0}=(3060-1)-27=3032 .
$$


As $M_{2}\left(\Gamma_{3}(2)\right)=\mathbf{1 5}_{a}$, a computation with $\operatorname{Sp}(6)$-representations shows that

$$
\begin{aligned}
\operatorname{Sym}^{4}\left(M_{2}\left(\Gamma_{3}(2)\right)\right) \cong & 2 \cdot \mathbf{1}+2 \cdot \mathbf{1 5}_{a}+\mathbf{2 7}_{a}+\mathbf{3 5}_{a}+4 \cdot \mathbf{3 5}_{b}+4 \cdot \mathbf{8} \mathbf{4}_{a}+\mathbf{1 0 5}_{c} \\
& +\mathbf{1 6 8}_{a}+\mathbf{1 8 9}_{c}+2 \cdot \mathbf{2 1 6}+3 \cdot \mathbf{2 8 0}_{b}+\mathbf{3 3 6}_{a}+\mathbf{4 2 0}_{a} .
\end{aligned}
$$

The $(1+27)$-dimensional kernel of the map $\operatorname{Sym}^{4}\left(M_{2}\left(\Gamma_{3}(2)\right)\right) \rightarrow$ $\operatorname{Sym}^{4}\left(M_{2}\left(\Gamma_{3}(2)\right)\right)_{0}$ must be an $\operatorname{Sp}(6)$-representation, thus it must be $\mathbf{1}+\mathbf{2 7}_{a}$. We have an explicit description of this kernel and we will show it is useful in the study of modular forms of higher genus in another paper.

It remains to identify the complementary $\mathrm{Sp}(6)$-representation, which we will denote by $W$ :

$$
M_{8}\left(\Gamma_{3}(2)\right) \cong \operatorname{Sym}^{4}\left(M_{2}\left(\Gamma_{3}(2)\right)\right)_{0} \oplus W, \quad \operatorname{dim} W=960 .
$$

We computed the operator $C$ from Section 3.8 on $\mathbf{C}\left[\ldots, X_{\sigma}, \ldots\right]_{16}^{H_{g}}$. The resulting pairs of the eigenvalues $\lambda$ with multiplicity $m_{\lambda}$ of $C$ are:

$$
\begin{array}{rllll}
\left(\lambda, m_{\lambda}\right): & (3,1050), & (9,840), \quad(15,168), & (27,140), \quad(63,2), \\
& (-3,672), & (-7,648), \quad(-9,35), & (-13,378), \quad(-21,60) .
\end{array}
$$

Note that this result also implies that $\mathbf{2 7}_{a}$ cannot be a subrepresentation of $\mathbf{C}\left[\ldots, X_{\sigma}, \ldots\right]_{16}^{H_{g}}$ because the eigenvalue $\lambda$ of $C$ on $\mathbf{2 7}_{a}$ would have been 35, but this is not an eigenvalue of $C$.

The eigenvalue 63 corresponds to the subspace of invariants. The eigenvalues $\lambda=9,-3,-7,-13$ occur only on the irreducible representations $\mathbf{2 8 0} b, \mathbf{3 3 6}_{a}, \mathbf{2 1 6}_{a}, \mathbf{1 8 9}_{c}$ respectively, hence $3 \cdot \mathbf{2 8 0}_{b}+2 \cdot \mathbf{3 3 6}_{a}+$ $3 \cdot \mathbf{2 1 6}_{a}+2 \cdot \mathbf{1 8 9}_{c}$ is a summand of $M_{8}\left(\Gamma_{3}(2)\right)$. Thus $W$ has a summand $189_{c}+216_{a}+336_{a}$.

The eigenvalue 3 occurs only on $\mathbf{8 4}, \mathbf{1 0 5}_{c}, \mathbf{2 1 0}_{b}$ and $\mathbf{4 2 0}$. As $4 \cdot \mathbf{8 4}_{a}+\mathbf{1 0 5}_{c}+\mathbf{4 2 0}_{a}$ is a summand of $\operatorname{Sym}^{4}\left(M_{2}\left(\Gamma_{3}(2)\right)\right)_{0}$, there remains a subrepresentation of dimension $1050-861=189$ in $W$ with this eigenvalue. That implies that $\mathbf{8 4} \mathbf{4}_{a}+\mathbf{1 0 5}_{c}$ is a summand of $W$.

The eigenvalue 15 occurs only on $\mathbf{1 0 5}_{b}, \mathbf{1 6 8}_{a}$ and $\mathbf{2 1 0} a$, as the eigenspace of $C$ for this eigenvalue has dimension 168 we conclude that $\mathbf{1 6 8}_{a}$ is a summand of $M_{8}\left(\Gamma_{3}(2)\right)$ (which lies in $\operatorname{Sym}^{4}\left(\mathbf{1 5}_{a}\right)$ ).

The eigenvalue 27 occurs only on $\mathbf{2 1}_{a}$ and $\mathbf{3 5}_{b}$. As $4 \cdot \mathbf{3 5}_{b}$ is a summand of $\operatorname{Sym}^{4}\left(M_{2}\left(\Gamma_{3}(2)\right)\right)_{0}$ and the dimension of this eigenspace of $C$ is 140 , none of these two representations occurs in $W$. 
The eigenvalue -9 occurs on four irreducible representations, the one with lowest dimension is $\mathbf{3 5} \mathbf{5}_{a}$. As the eigenspace for $\lambda=-9$ has dimension 35, we conclude that $\mathbf{3 5}_{a}$ is a summand of $M_{8}\left(\Gamma_{3}(2)\right.$ ) (which lies in $\operatorname{Sym}^{4}\left(\mathbf{1 5}_{a}\right)$ ).

The eigenvalue -21 occurs only on $\mathbf{1 5}{ }_{a}$ and $\mathbf{1 0 5}$, as the eigenspace of $C$ for this eigenvalue has dimension 60 we conclude that $4 \cdot \mathbf{1 5}_{a}$ is a summand of $M_{8}\left(\Gamma_{3}(2)\right)$ and that $W$ has a summand $2 \cdot \mathbf{1 5}_{a}$.

From this we find the following decomposition of $W$ :

$$
W=2 \cdot \mathbf{1 5} 5_{a}+\mathbf{8 4} 4_{a}+\mathbf{1 0 5} 5_{c}+\mathbf{1 8 9} 9_{c}+\mathbf{2 1 6} 6_{a}+\mathbf{3 3 6} 6_{a} .
$$

\section{$7 \quad$ The case $g=4$}

\subsection{The $\operatorname{Sp}(8)$-representation on $M_{4}^{\theta}\left(\Gamma_{4}(2)\right)$}

In case $g=4$ we are no longer sure if $M_{2 k}^{\theta}\left(\Gamma_{4}(2)\right)$, the space of modular forms of weight of $2 k$ which are (Heisenberg-invariant) polynomials in the $\Theta[\sigma]$ 's, is equal to all of $M_{2 k}\left(\Gamma_{4}(2)\right)$ (cf. [22]).

The $\operatorname{Sp}(8)$-representation on $M_{2}^{\theta}\left(\Gamma_{4}(2)\right)$, which we denoted by $\rho_{\theta}$ in Section 3.6, is the unique 51-dimensional irreducible representation of $\operatorname{Sp}(8)$ (a table of the 81 irreducible representations of $\mathrm{Sp}(8)$ can be easily generated with the computer algebra program 'Magma' [2]).

The complement of $\operatorname{Sym}^{2}\left(M_{2}^{\theta}\left(\Gamma_{4}(2)\right)\right.$ in $M_{4}^{\theta}\left(\Gamma_{4}(2)\right)$ (of $\operatorname{Sp}(8)$ representations) now has codimension 918 :

$$
\begin{aligned}
\operatorname{dim} & M_{4}^{\theta}\left(\Gamma_{4}(2)\right)-\operatorname{dim} \operatorname{Sym}^{2}\left(M_{2}^{\theta}\left(\Gamma_{4}(2)\right)\right) \\
= & 2244-\left(\begin{array}{c}
51+1 \\
2
\end{array}\right)=2244-1326=918 .
\end{aligned}
$$

We computed the operator $C$ from Section 3.8 on $M_{4}^{\theta}\left(\Gamma_{4}(2)\right)$. The resulting pairs of its eigenvalues $\lambda$ with multiplicity $m_{\lambda}$ are

$$
\left(\lambda, m_{\lambda}\right): \quad(-25,918), \quad(39,1190), \quad(119,135), \quad(255,1) .
$$

The last three eigenspaces of $C$ correspond to the irreducible representations $\sigma_{c}, \sigma_{\theta}$ and 1 respectively and their direct sum is $\operatorname{Sym}^{2}\left(\rho_{\theta}\right)$, cf. Section 3.7. The character table shows that there are only 10 irreducible representations of $\mathrm{Sp}(8)$ with dimension less then 918 and there is a unique irreducible representation with dimension 918. However, of these eleven irreducible representations, the map $C$ has eigenvalue $\lambda=-25$ only on the one of dimension 
918. Thus we conclude that $M_{4}^{\theta}\left(\Gamma_{4}(2)\right)$ is the sum of just four irreducible representations (like $M_{4}^{\theta}\left(\Gamma_{3}(2)\right.$ ), cf. Section 6.4).

\subsection{Frame's observations on $\operatorname{Sp}(2 g)$-representations}

Frame [11] observed that the irreducible representations of $\operatorname{Sp}(2 g)$ seem to come in series, parametrized by $g$ and an irreducible representation of $O^{+}(2 l)$ or $O^{-}(2 l)$ for some $l<g$, he called the integer $l$ the level of the representation.

The dimension of an irreducible representation of level $l$, as function of $g$, should be a polynomial in $2^{g}$ of degree $2 l$. For example, the representations $\rho_{\theta}$ (of dimension $\left.\left(2^{g}+1\right)\left(2^{g-1}+1\right) / 3=\left(2^{g}+1\right)\left(2^{g}+2\right) /(3 !)\right)$ is of level one and should correspond to a representation of $O^{-}(2) \cong S_{3}$.

Note that we have:

$$
\begin{aligned}
\operatorname{dim} & M_{4}^{\theta}\left(\Gamma_{g}(2)\right)-\operatorname{dim} \operatorname{Sym}^{2}\left(M_{2}^{\theta}\left(\Gamma_{g}(2)\right)\right) \\
= & \frac{1}{8 !}\left(2^{g}+1\right)\left(2^{g}-1\right)\left(2^{g}+2\right)\left(2^{g}-2\right)\left(2^{g}-2^{2}\right)\left(2^{g}+2^{5}\right)
\end{aligned}
$$

which suggests, in this frame work, that $M_{4}^{\theta}\left(\Gamma_{g}(2)\right)$ is the direct sum of $\operatorname{Sym}^{2}\left(M_{2}^{\theta}\left(\Gamma_{g}(2)\right)\right)$ (itself a direct sum of three irreducible representations with multiplicity one) and an irreducible representation of level 3 corresponding to a representation of $O^{+}(6) \cong S_{8}$, generalizing the results in Sections 6.4 and 7.1 .

\section{Applications to superstring measures}

\subsection{Introduction}

In this section we consider applications of our results to modular forms $\Xi_{6}\left[0^{(g)}\right]$ and $\Xi_{8}\left[0^{(g)}\right]$ on $\Gamma_{g}(2)$, of weight 6 and 8 , respectively, which appear to play an important role in the theory of superstrings (cf. $[4,6-8,19])$. One has $\Xi_{6}\left[0^{(1)}\right]=\eta^{12}$ and $\Xi_{8}\left[0^{(1)}\right]=\theta\left[\begin{array}{l}0 \\ 0\end{array}\right]^{4} \eta^{12}$. In case $g=2$ D'Hoker and Phong determined, using superstring theory, a modular form $\Xi_{6}\left[0^{(2)}\right] \in M_{6}\left(\Gamma_{2}(2)\right)^{\epsilon}$.

As it is (still) difficult to extend their methods to higher genera, they stated certain constraints which should be satisfied by the $\Xi_{6}\left[0^{(g)}\right]$. However, in genus three they could not find a modular form satisfying their constraints and we show in 8.3 that indeed such a modular form does not exist. 
In [4] the constraints were modified and now one has to find $\mathrm{O}^{+}$-invariant modular forms $\Xi_{8}\left[0^{(g)}\right]$ of weight 8 on $\Gamma_{g}(2)$ which moreover restrict to $\Xi_{8}\left[0^{(k)}\right] \Xi_{8}\left[0^{(g-k)}\right]$ on the subvarieties $\mathbf{H}_{k} \times \mathbf{H}_{g-k} \subset \mathbf{H}_{g}$. Actually, superstring theory only requires the $\Xi_{8}$ 's to be defined on the Jacobi locus $J_{g}$ of period matrices of Riemann surfaces.

In $[4,5]$ modular forms $\Xi_{8}\left[0^{(3)}\right]$ and $\Xi_{8}\left[0^{(4)}\right]$ satisfying the modified constraints were found. Grushevsky [14] gives a more general approach for all genera but it is not clear that his proposal leads to single valued functions for $g \geq 6$, for $g=5$ see [26]. Here we give uniqueness results for these functions. For example in genus two the function $\Xi_{6}\left[0^{(2)}\right]$, found with hard work by D'Hoker and Phong, is easily recovered as the only modular form satisfying their constraints (but the validity in superstring theory of the (modified) constraints is not clear yet).

\subsection{The uniqueness of $\Xi_{8}\left[0^{(2)}\right]$}

In $\left[4\right.$, Section 3.4], we announced that a certain modular form $\Xi_{8}\left[0^{(2)}\right] \in$ $M_{8}\left(\Gamma_{2}(2)\right)^{O^{+}}$, that is, a modular form on $\Gamma_{g}(1,2)$, is the unique modular form satisfying certain constraints. To verify this, we recall from 3.5 that $\operatorname{dim} M_{8}\left(\Gamma_{2}(2)\right)^{O^{+}}=n_{1}+n_{\theta}$, with $n_{1}, n_{\theta}$ the multiplicity of $\mathbf{1}$ and $\sigma_{\theta}=\rho_{[42]}$ in $M_{8}\left(\Gamma_{2}(2)\right)$, respectively, see Section 5.2. From the decomposition given there we find that

$$
\operatorname{dim} M_{8}\left(\Gamma_{2}(2)\right)^{O^{+}}=1+3=4
$$

The subspace $M_{8}\left(\Gamma_{2}(2)\right)^{O^{+}}$contains the $\operatorname{Sp}(4)$-invariant $\sum_{\delta} \theta[\delta]^{16} \in$ $M_{8}\left(\Gamma_{2}(2)\right)$ as well as the three dimensional subspace spanned by

$$
f_{1}=\theta\left[\begin{array}{l}
00 \\
00
\end{array}\right]^{16}, \quad f_{2}=\theta\left[\begin{array}{l}
00 \\
00
\end{array}\right]^{4} \sum_{\delta} \theta[\delta]^{12}, \quad f_{3}=\theta\left[\begin{array}{l}
00 \\
00
\end{array}\right]^{8} \sum_{\delta} \theta[\delta]^{8} .
$$

We checked, using the classical theta formulas, that these four functions are linearly independent and thus are a basis of $M_{8}\left(\Gamma_{2}(2)\right)^{O^{+}}$.

The function $\Xi_{8}\left[0^{(2)}\right]$ should restrict to $\Xi_{8}\left[0^{(1)}\right]\left(\tau_{1}\right) \Xi_{8}\left[0^{(1)}\right]\left(\tau_{2}\right)$ with $\Xi_{8}\left[0^{(1)}\right]\left(\tau_{1}\right)=\left(\theta\left[{ }_{0}^{0}\right]^{4} \eta^{12}\right)\left(\tau_{1}\right)$ on $\mathbf{H}_{1} \times \mathbf{H}_{1} \subset \mathbf{H}_{2}$. This function is a multiple of $\theta\left[\begin{array}{l}0 \\ 0\end{array}\right]^{4}\left(\tau_{1}\right)$. The restrictions of the $f_{i}$ are also multiples of $\theta\left[\begin{array}{l}0 \\ 0\end{array}\right]^{4}\left(\tau_{1}\right)$, but the restriction of $\sum \theta[\delta]^{16}$ is not. Hence $\Xi_{8}\left[0^{(2)}\right]$ should be linear combination of the three $f_{i}$, and in [4] we showed that there is a unique such linear combination satisfying all the constraints. Hence $\Xi_{8}\left[0^{(2)}\right]$ is unique. Moreover, 
the formula given in [4] shows that $\Xi_{8}\left[0^{(2)}\right]=\theta\left[0^{(2)}\right]^{4} \Xi_{6}\left[0^{(2)}\right]$ where $\Xi_{6}\left[0^{(2)}\right]$ is the modular form found by D'Hoker and Phong.

\subsection{The non-existence of $\Xi_{6}\left[0^{(3)}\right]$}

In [8] the existence of a modular form $\Xi_{6}\left[0^{(3)}\right] \in M_{6}\left(\Gamma_{g}(2)\right)^{\epsilon}$ with certain properties is investigated. The only $\operatorname{Sp}(6)$-representations with $\mathrm{O}^{+}$-antiinvariants are $\rho_{\theta}=\mathbf{1 5}_{a}$ and $\rho_{r}=\mathbf{2 1}_{b}$ and these have a unique such antiinvariant (cf. Section 3.5). Thus from the decomposition of $M_{6}\left(\Gamma_{3}(2)\right.$ ) given in Section 6.5 it follows that $\operatorname{dim} M_{6}\left(\Gamma_{3}(2)\right)^{\epsilon}=3$. We verified that the following functions are a basis:

$$
M_{6}\left(\Gamma_{3}(2)\right)^{\epsilon}=\left\langle\theta\left[\begin{array}{c}
{[000} \\
000
\end{array}\right]^{12}, \quad \sum_{\Delta} \theta[\Delta]^{12}, \quad \theta\left[\begin{array}{l}
0000 \\
000
\end{array}\right]^{4} \sum_{\Delta} \theta[\Delta]^{8}\right\rangle
$$

The function $\Xi_{6}\left[0^{(3)}\right]$ should restrict to $\Xi_{6}\left[0^{(1)}\right]\left(\tau_{1}\right) \Xi_{6}\left[0^{(2)}\right]\left(\tau_{2}\right)$ for $\left(\tau_{1}, \tau_{2}\right) \in$ $\mathbf{H}_{1} \times \mathbf{H}_{2} \subset \mathbf{H}_{3}$, with $\Xi_{6}\left[0^{(1)}\right]\left(\tau_{1}\right)=\eta^{12}\left(\tau_{1}\right)$. The restriction map on the theta constants is given by

$$
\theta\left[\begin{array}{c}
a b c \\
d e f
\end{array}\right](\tau) \longmapsto \theta\left[\begin{array}{c}
a \\
d
\end{array}\right]\left(\tau_{1}\right) \theta\left[\begin{array}{c}
b c \\
e f
\end{array}\right]\left(\tau_{2}\right),
$$

in particular $\theta\left[\begin{array}{l}a b c \\ d e f\end{array}\right] \mapsto 0$ if $a d=1$. Thus six of the 36 even theta constants map to zero, the other $30=3 \cdot 10$ are uniquely decomposed in the product of two even theta constants for $g=1$ and $g=2$, respectively. Hence, using the results from Section 4.4 and [4], we get

$$
\begin{aligned}
\theta\left[\begin{array}{l}
000 \\
000
\end{array}\right]^{12}\left(\tau_{1,2}\right) & =\theta\left[\begin{array}{l}
0 \\
0
\end{array}\right]^{12}\left(\tau_{1}\right) \theta\left[\begin{array}{l}
00 \\
00
\end{array}\right]^{12}\left(\tau_{2}\right) \\
& =\left(\frac{1}{3} f_{21}+\eta^{12}\right) \theta\left[\begin{array}{l}
00 \\
00
\end{array}\right]^{12}, \\
\left(\sum_{\Delta} \theta[\Delta]^{12}\right)\left(\tau_{1,2}\right) & =\left(\theta\left[\begin{array}{l}
0 \\
0
\end{array}\right]^{12}+\theta\left[\begin{array}{l}
0 \\
1
\end{array}\right]^{12}+\theta\left[\begin{array}{l}
1 \\
0
\end{array}\right]^{12}\right)\left(\tau_{1}\right)\left(\sum_{\delta} \theta[\delta]^{12}\right)\left(\tau_{2}\right) \\
& =\left(\frac{2}{3} f_{21}-\eta^{12}\right) \sum_{\delta} \theta[\delta]^{12} \\
\left(\theta\left[\begin{array}{l}
000 \\
000
\end{array}\right]^{4} \Psi_{4}\right)\left(\tau_{1,2}\right) & =\theta\left[\begin{array}{l}
000 \\
000
\end{array}\right]^{4}\left(\tau_{1,2}\right)\left(\sum_{\Delta} \theta[\Delta]^{8}\right)\left(\tau_{1,2}\right) \\
& =\theta\left[\begin{array}{l}
0 \\
0
\end{array}\right]^{4}\left(\tau_{1}\right)\left(\theta\left[\begin{array}{l}
0 \\
0
\end{array}\right]^{8}+\theta\left[\begin{array}{l}
0 \\
1
\end{array}\right]^{8}+\theta\left[\begin{array}{l}
1 \\
0
\end{array}\right]^{8}\right)\left(\tau_{1}\right) \theta\left[\begin{array}{l}
00 \\
00
\end{array}\right]^{4}\left(\tau_{2}\right)
\end{aligned}
$$




$$
\begin{aligned}
& \times\left(\sum_{\delta} \theta[\delta]^{8}\right)\left(\tau_{2}\right) \\
= & \left.\frac{2}{3} f_{21} \theta[00]^{0}\right]^{4}\left(\sum_{\delta} \theta[\delta]^{8}\right) .
\end{aligned}
$$

Thus $a \theta\left[\begin{array}{l}000 \\ 000\end{array}\right]^{12}+b \sum_{\Delta} \theta[\Delta]^{12}+c \theta[000]^{000}{ }^{4}\left(\sum_{\Delta} \theta[\Delta]^{8}\right)$ restricts to a function of the form $\eta^{12}\left(\tau_{1}\right) g\left(\tau_{2}\right)$ iff

$$
a \theta\left[\begin{array}{l}
00 \\
00
\end{array}\right]^{12}+2 b \sum_{\delta} \theta[\delta]^{12}+2 c \theta\left[\begin{array}{l}
00 \\
00
\end{array}\right]^{4} \sum_{\delta} \theta[\delta]^{8}=0
$$

on $\mathbf{H}_{2}$. However, it follows from the results in Table 6 of [3] that the ten even $\theta[\delta]^{12}$ 's span a ten dimensional space and that $\theta\left[\begin{array}{l}00 \\ 00\end{array}\right]^{4} \sum_{\delta} \theta[\delta]^{8}$ does not lie in that 10-dimensional space. Hence we must have $a=b=c=0$, which proves that there is no function $\Xi_{6}\left[0^{(3)}\right]$ satisfying the three constraints imposed in $[8]$.

\subsection{The uniqueness of $\Xi_{8}\left[0^{(3)}\right]$}

In [4] we found a modular form $\Xi_{8}\left[\begin{array}{l}000 \\ 000\end{array}\right]$ which satisfied constraints similar to those of [8] and which are expected to be related to the superstring measure.

The constraints imply that $\Xi_{8}\left[0^{(3)}\right]$ is a modular form on $\Gamma_{3}(2)$ of weight 8 and should be $\mathrm{O}^{+}$-invariant (equivalently, it is a modular form on $\Gamma_{3}(1,2)$ ), so it must lie in $M_{8}\left(\Gamma_{3}(2)\right)^{O^{+}}$. The only $\mathrm{Sp}(6)$-representations that have $O^{+}(6)$-invariants are $\mathbf{1}$ and $\sigma_{\theta}=\mathbf{3 5}_{b}$. Hence (cf. Section 3.5), the decomposition of $M_{8}\left(\Gamma_{3}(2)\right)$ obtained in Section 6.10 implies that

$$
\operatorname{dim} M_{8}\left(\Gamma_{3}(2)\right)^{O^{+}}=1+4=5 .
$$

The subspace $M_{8}\left(\Gamma_{3}(2)\right)^{O^{+}}$contains the $\operatorname{Sp}(6)$-invariant $\sum_{\Delta} \theta[\Delta]^{16} \in$ $M_{8}\left(\Gamma_{3}(2)\right)$ as well as the three dimensional subspace

$$
\theta\left[\begin{array}{l}
000 \\
000
\end{array}\right]^{4} M_{6}\left(\Gamma_{3}(2)\right)^{\epsilon}:=\left\{\theta\left[\begin{array}{l}
000 \\
000
\end{array}\right]^{4} f: f \in M_{6}\left(\Gamma_{3}(2)\right)^{\epsilon}\right\},
$$

since both $\theta\left[\begin{array}{l}000 \\ 000\end{array}\right]^{4}$ and such $f$ are $O^{+}$-anti-invariant.

For a Lagrangian subspace $L \subset \mathbf{F}_{2}^{6}$ we defined modular forms $\pm P_{L} \in$ $M_{4}\left(\Gamma_{3}(2)\right.$ ) (cf. Section 6.4). For an even characteristic $\Delta$, the sum of the 
$30 P_{L}^{2}$ 's such that $L \subset Q_{\Delta}$, where $Q_{\Delta}$ is the quadric in $\mathbf{F}_{2}^{6}$ defined by $\Delta$ is a modular form of weight 8 on $\Gamma_{g}(2)$ :

$$
G[\Delta]:=\sum_{L \subset Q_{\Delta}} P_{L}^{2}=\sum_{L \subset Q_{\Delta}} \prod_{Q^{\prime} \supset L} \theta\left[\Delta_{Q^{\prime}}\right]^{2} \quad\left(\in M_{8}\left(\Gamma_{3}(2)\right)\right) .
$$

Note that $\theta[\Delta]^{2}$ is one of the factors in each of the 30 products. In [4] we verified that $G[0] \in M_{8}\left(\Gamma_{3}(2)\right)^{O^{+}}$.

It is not hard to verify (using the classical theta formulas for example) that we now have functions which span the $\mathrm{O}^{+}$-invariants:

$$
M_{8}\left(\Gamma_{4}(2)\right)^{O^{+}}=\theta\left[\begin{array}{c}
000 \\
000
\end{array}\right]^{4} M_{6}\left(\Gamma_{3}(2)\right)^{\epsilon} \oplus\left\langle\sum_{\Delta} \theta[\Delta]^{16}, G[0]\right\rangle .
$$

The modular form $\Xi_{8}\left[0^{(0)}\right]$ should moreover restrict to $\left(\theta\left[\begin{array}{l}0 \\ 0\end{array}\right]^{4} \eta^{12}\right)\left(\tau_{1}\right)\left(\theta\left[\begin{array}{l}00 \\ 00\end{array}\right]^{4} \Xi_{6}\left[\begin{array}{l}00 \\ 00\end{array}\right]\right)\left(\tau_{2}\right)$ on $\mathbf{H}_{1} \times \mathbf{H}_{2} \subset \mathbf{H}_{3}$, note this restriction is a multiple of $\theta\left[\begin{array}{l}0 \\ 0\end{array}\right]\left(\tau_{1}\right)$.

The restriction of $\sum \theta[\Delta]^{16}$ to the diagonal is not a multiple of $\theta\left[{ }_{0}^{0}\right]\left(\tau_{1}\right)$, whereas the other four functions do have a restriction which is a multiple of $\theta\left[\begin{array}{l}0 \\ 0\end{array}\right]\left(\tau_{1}\right)$. Thus $\Xi_{8}\left[0^{(3)}\right]$ should be linear combination

$$
\Xi_{a, b, c, d}:=\theta\left[\begin{array}{l}
{[000} \\
000
\end{array}\right]^{4}\left(a \theta\left[\begin{array}{c}
{[000} \\
000
\end{array}\right]^{12}+b \sum_{\Delta} \theta[\Delta]^{12}+c \theta\left[\begin{array}{l}
{[000} \\
000
\end{array}\right]^{4} \Psi_{4}\right)+d G\left[\begin{array}{l}
000 \\
000
\end{array}\right] .
$$

An explicit computation (cf. [4]) shows that only $\Xi_{8}\left[0^{(3)}\right]:=\Xi_{4,4,-3,-12} / 12$ restricts to $\left(\theta\left[\begin{array}{l}0 \\ 0\end{array}\right]^{4} \eta^{12}\right)\left(\tau_{1}\right)\left(\theta\left[\begin{array}{l}00 \\ 00\end{array}\right]^{4} \Xi_{6}\left[\begin{array}{l}00 \\ 00\end{array}\right]\right)\left(\tau_{2}\right)$. This verifies the uniqueness of $\Xi_{8}\left[0^{(3)}\right]$.

\subsection{The uniqueness of $\Xi_{8}\left[0^{(4)}\right]$}

In $[5,14]$ a modular form $\Xi_{8}\left[0^{(4)}\right] \in M_{8}\left(\Gamma_{4}(2)\right)^{O^{+}}$was defined which restricts to $\Xi_{8}\left[0^{(a)}\right]\left(\tau_{a}\right) \Xi_{8}\left[0^{(b)}\right]\left(\tau_{b}\right)$ for $\left(\tau_{a}, \tau_{b}\right) \in \mathbf{H}_{a} \times \mathbf{H}_{b} \subset \mathbf{H}_{4}$ for $(a, b)=$ $(1,3),(2,2)$. We will assume from now on that $\Xi_{8}\left[0^{(4)}\right]$ actually is in $M_{8}^{\theta}\left(\Gamma_{4}(2)\right)^{O^{+}}$(this is currently under investigation).

Using the same methods as in Sections 8.2 and 8.4, one can show that these properties characterize $\Xi_{8}\left[0^{(4)}\right]$, up to adding a term $\lambda J$, where $J=0$ defines the Jacobi locus $J_{4}$ (the locus of period matrices of Riemann surfaces in $\mathbf{H}_{4}$ ) and $\lambda \in \mathbf{C}$. In fact, $J$ is a modular form of weight 8 on $\Gamma_{4}$, so it 
can be added to $\Xi_{8}\left[0^{(4)}\right]$ without changing its $\Gamma_{g}(1,2)$-invariance, moreover $\mathbf{H}_{1} \times \mathbf{H}_{3}$ and $\mathbf{H}_{2} \times \mathbf{H}_{2}$ are contained in the closure of the Jacobi locus, so $J$ is zero on these loci.

The key point is the determination of the dimension of $M_{8}^{\theta}\left(\Gamma_{4}(2)\right)^{O^{+}}$, which could be done by computer. Recently Oura [21] determined this dimension using the methods from [23, 24]: $\operatorname{dim} M_{8}^{\theta}\left(\Gamma_{4}(2)\right)^{O^{+}}=7$. As we already know seven independent functions in $M_{8}^{\theta}\left(\Gamma_{4}(2)\right)^{O^{+}}$, it follows (using the notation from [5]):

$$
\begin{aligned}
M_{8}^{\theta}\left(\Gamma_{4}(2)\right)^{O^{+}}= & \left\langle\sum_{\Delta} \theta[\Delta]^{16},\left(\sum_{\Delta} \theta[\Delta]^{8}\right)^{2}, \theta\left[0^{(4)}\right] \tilde{F}_{1}, \theta\left[0^{(4)}\right] \tilde{F}_{2}, \theta\left[0^{(4)}\right] \tilde{F}_{3},\right. \\
& \left.G_{1}\left[0^{(4)}\right], G_{2}\left[0^{(4)}\right]\right\rangle .
\end{aligned}
$$

The modular form $J$ vanishing on the Jacobi locus is

$$
J=16 \sum \theta[\Delta]^{16}-\left(\sum \theta[\Delta]^{8}\right)^{2}
$$

(cf. [17]). Now the proof of the unicity of $\Xi_{8}\left[0^{(4)}\right]$ in $M_{8}^{\theta}\left(\Gamma_{g}(2)\right)$ can be obtained with arguments similar to those in Sections 8.2 and 8.4.

\subsection{The cosmological constant in $g=3$ and $g=4$}

In [4, Section 4.5] we showed that the cosmological constant, which is the sum over the 36 even characteristics of $\Xi_{8}\left[\Delta^{(3)}\right]$, is zero. We used that there is a unique, up to scalar multiple, $\mathrm{Sp}(6)$-invariant in $M_{8}\left(\Gamma_{3}(2)\right)$. This follows directly from the fact that in the decomposition of $M_{8}\left(\Gamma_{3}(2)\right)$ the representation $\mathbf{1}$ has multiplicity one.

In case $g=4$, Grushevsky [14] already proved that the cosmological constant vanishes using results on the slope of divisors on the moduli space of genus four curves. Another proof was given by Salvati Manni [26]. We sketch an alternative approach to this result, similar to the $g=3$ case in [4]. A computer computation showed that the space of $\operatorname{Sp}(8)$-invariants in $\mathbf{C}\left[\ldots, X_{\sigma}, \ldots\right]_{16}^{H_{g}}$ is two dimensional (one starts with finding invariants for the transvections which act 'diagonally' on the $X_{\sigma}$ 's to reduce the computation to a manageable size). These invariants correspond to the modular forms $\Psi_{8}, \Psi_{4}^{2}$ with $\Psi_{4 k}(\tau):=\sum \theta\left[\Delta^{(4)}\right]^{8 k}$, note that $J=16 \Psi_{8}-\Psi_{4}^{2}$ is zero on the Jacobi locus. 
As $\sum_{\Delta} \Xi_{8}\left[\Delta^{(4)}\right]$ is an $\operatorname{Sp}(8)$-invariant of weight 8 , there are constants $\lambda, \mu$ such that

$$
\sum_{\Delta} \Xi_{8}\left[\Delta^{(4)}\right]=\lambda \Psi_{8}+\mu \Psi_{4}^{2}
$$

and it suffices to show that $\lambda+16 \mu=0$. For this one can specialize $\tau$ to a diagonal matrix $\tau=\operatorname{diag}\left(\tau_{1}, \ldots, \tau_{4}\right)$ and then let $\tau_{j} \mapsto \infty$ for $j=1, \ldots, 4$, similar to the computation in [4, Section 4.5].

\section{Acknowledgments}

We would like to thank Sergio L. Cacciatori for several interesting and stimulating discussions, R. Salvati Manni for useful discussions and references and to L. Paredi for supporting us in computer computations.

\section{Appendix A The action of $\operatorname{Sp}(2 g)$ on the Heisenberg invariants}

\section{A.1 Characteristics and quadrics}

The group $\operatorname{Sp}(2 g)$ fixes a non-degenerate alternating form $E$ (i.e. $E(v, v)=0$ for all $v$ ) on the $\mathbf{F}_{2}$-vector space $V=\mathbf{F}_{2}^{2 g}$. We choose a symplectic basis of $V$ so that

$$
\begin{aligned}
& E: V \times V \longrightarrow \mathbf{F}_{2}, \\
& E(v, w):=v_{1} w_{g+1}+\cdots+v_{g} w_{2 g}+v_{g+1} w_{1}+\cdots+v_{2 g} w_{g},
\end{aligned}
$$

or, more compactly, $E\left(\left(v^{\prime}, v^{\prime \prime}\right),\left(w^{\prime}, w^{\prime \prime}\right)\right)={ }^{t} v^{\prime} w^{\prime \prime}+{ }^{t} v^{\prime \prime} w^{\prime}$ and we occasionally write $v=\left(\begin{array}{l}v^{\prime} \\ v^{\prime \prime}\end{array}\right)$, where $v^{\prime}, v^{\prime \prime}$ are then considered as row vectors.

We consider the quadratic forms $q$ on $V$ whose associated bilinear form is $E$ (see also [4, Appendix A], [16, V.6]), that is the maps

$$
q: V \longrightarrow \mathbf{F}_{2}, \quad q(v+w)=q(v)+q(w)+E(v, w) .
$$

One verifies easily that for all $a_{i}, b_{i} \in \mathbf{F}_{2}$ the function

$$
\begin{aligned}
q(v)= & v_{1} v_{g+1}+v_{2} v_{g+2}+\cdots+v_{g} v_{2 g}+a_{1} v_{1}+\cdots+a_{g} v_{g} \\
& +b_{1} v_{g+1}+\cdots+b_{g} v_{2 g}
\end{aligned}
$$

satisfies $q(v+w)=q(v)+q(w)+E(v, w)$ and that any quadratic form associated to $E$ is of this form. Note that $q(v)={ }^{t} v^{\prime} v^{\prime \prime}+a v^{\prime}+b v^{\prime \prime}$, with row 
vectors $a=\left(a_{1}, \ldots, a_{g}\right), b=\left(b_{1}, \ldots, b_{g}\right)$. The (theta) characteristic $\Delta_{q}$ associated to $q$ is defined as

$$
\Delta_{q}:=\left[\begin{array}{l}
a_{1} a_{2} \ldots a_{g} \\
b_{1} b_{2} \ldots b_{g}
\end{array}\right]=\left[\begin{array}{l}
a \\
b
\end{array}\right], \quad \text { let } \quad e\left(\Delta_{q}\right):=(-1)^{\sum_{i=1}^{g} a_{i} b_{i}} \quad(\in\{1,-1\}) .
$$

We say that $\Delta_{q}$ is even if $e\left(\Delta_{q}\right)=+1$ and odd else. One can verify that $q(v)$ has $2^{g-1}\left(2^{g}+1\right)$ zeroes in $V$ if $\Delta_{q}$ is even and has $2^{g-1}\left(2^{g}-1\right)$ zeroes if $\Delta_{q}$ is odd. Moreover, there are $2^{g-1}\left(2^{g}+1\right)$ even characteristics and $2^{g-1}\left(2^{g}-\right.$ 1 ) odd characteristics, thus there are $3,10,36,136$ even characteristics and $1,6,28,120$ odd characteristics for $g=1,2,3,4$, respectively.

The group $\operatorname{Sp}(2 g)$ acts naturally on the characteristics by

$$
(g \cdot q)(v):=q\left(g^{-1} v\right) \quad(g \in \operatorname{Sp}(2 g), v \in V) .
$$

This action is transitive (actually doubly transitive [16, V.6, Corollary]) on both the set of even characteristic and on the set of odd characteristics.

\section{A.2 Theta constants with characteristics}

For an even characteristic $\Delta=\left[\begin{array}{l}a \\ b\end{array}\right]$ one defines a function, a theta constant, on the Siegel space $\mathbf{H}_{g}$ by

$$
\theta\left[\begin{array}{l}
a \\
b
\end{array}\right](\tau):=\sum_{m \in \mathbf{Z}^{g}} \mathrm{e}^{\pi \mathrm{i}\left((m+a / 2) \tau^{t}(m+a / 2)+(m+a / 2)^{t} b\right)}
$$

so $m$ is a row vector and $\sum a_{i} b_{i} \equiv 0 \bmod 2$. Then one has [16, V.1, Corollary], with $\kappa(M) \mathrm{e}^{2 \pi \mathrm{i} \phi_{\Delta}(M)}$ an eight-root of unity, that

$$
\theta[M \cdot \Delta](M \cdot \tau)=\kappa(M) \mathrm{e}^{2 \pi \mathrm{i} \phi_{\Delta}(M)} \operatorname{det}(C \tau+D)^{1 / 2} \theta[\Delta](\tau),
$$

for all $M \in \operatorname{Sp}(2 g, \mathbf{Z})$, here the action of $M$ on the characteristic $\Delta$ is given by

$$
\left(\begin{array}{ll}
A & B \\
C & D
\end{array}\right) \cdot\left[\begin{array}{l}
a \\
b
\end{array}\right]:=\left[\begin{array}{l}
c \\
d
\end{array}\right], \quad\left(\begin{array}{c}
{ }^{t} c \\
{ }^{t} d
\end{array}\right)=\left(\begin{array}{cc}
D & -C \\
-B & A
\end{array}\right)\left(\begin{array}{l}
{ }^{t} a \\
{ }^{t} b
\end{array}\right)+\left(\begin{array}{c}
t \\
{ }^{t}\left(C^{t} D\right)_{0} \\
{ }^{t}\left(A^{t} B\right)_{0}
\end{array}\right) \quad \bmod 2
$$

where $N_{0}=\left(N_{11}, \ldots, N_{g g}\right)$ is the row vector of diagonal entries of the matrix $N$. 
To get representations of (quotients of) $\Gamma_{g}$, we defined the action of $\Gamma_{g}$ on modular forms of weight $k$ by $(M \cdot f)(\tau):=\left(\operatorname{det}(C \tau+D)^{k} f\left(M^{-1} \cdot \tau\right)\right.$ (cf. Section 2.4). Note that

$$
\theta[\Delta]\left(M^{-1} \cdot \tau\right)=\theta\left[M^{-1} M \cdot \Delta\right]\left(M^{-1} \cdot \tau\right)=c_{M^{-1}, \Delta, \tau} \theta[M \cdot \Delta](\tau),
$$

where $c_{M^{-1, \Delta, \tau}}$ collects the non-relevant part. Thus the action of $M$ basically maps $\theta[\Delta]$ to $\theta[M \cdot \Delta]$.

\section{A.3 The action of $\operatorname{Sp}(2 g)$ on the characteristics}

We show explicitly that

$$
\left(M \cdot q_{\Delta}\right)(v)=q_{M \cdot \Delta}(v),
$$

which verifies that the natural action of $\Gamma_{g}$ on the theta characteristics corresponds to its action on the quadratic forms on $V$.

As $\left(M \cdot q_{\Delta}\right)(v)=q_{\Delta}\left(M^{-1} v\right)$, we must verify $q_{\Delta}\left(M^{-1} v\right)=q_{M \cdot \Delta}(v)$. For $M \in \Gamma_{g}=\operatorname{Sp}(2 g, \mathbf{Z})$ we have $M E^{t} M=E$ where $E$ has blocks $A=D=$ $0, B=-C=I$ :

$$
M E^{t} M=E \quad \text { iff } \quad\left(\begin{array}{ll}
-B^{t} A+A^{t} B & -B^{t} C+A^{t} D \\
-D^{t} A+C^{t} B & -D^{t} C+C^{t} D
\end{array}\right)=\left(\begin{array}{cc}
0 & I \\
-I & 0
\end{array}\right) .
$$

As $E^{-1}=-E$ we find that

$$
M^{-1}=-E^{t} M E, \quad \text { so } \quad M^{-1} v=\left(\begin{array}{cc}
{ }^{t} D & -{ }^{t} B \\
-{ }^{t} C & { }^{t} A
\end{array}\right)\left(\begin{array}{c}
v^{\prime} \\
v^{\prime \prime}
\end{array}\right)=\left(\begin{array}{c}
{ }^{t} D v^{\prime}-{ }^{t} B v^{\prime \prime} \\
-{ }^{t} C v^{\prime}+{ }^{t} A v^{\prime \prime}
\end{array}\right) .
$$

Therefore, with $\left.q_{\Delta}(v)=q_{[a}^{a}\right],\left(\left(\begin{array}{c}v^{\prime} \\ v^{\prime \prime}\end{array}\right)\right)=t^{\prime} v^{\prime \prime}+a v^{\prime}+b v^{\prime \prime}$, we get

$$
\begin{aligned}
q_{\Delta}\left(M^{-1} v\right)= & \left({ }^{t} D v^{\prime}-{ }^{t} B v^{\prime \prime}\right)\left(-{ }^{t} C v^{\prime}+{ }^{t} A v^{\prime \prime}\right) \\
& +a\left({ }^{t} D v^{\prime}-{ }^{t} B v^{\prime \prime}\right)+b\left(-{ }^{t} C v^{\prime}+{ }^{t} A v^{\prime \prime}\right) .
\end{aligned}
$$

The non-linear part is

$$
{ }^{t} v^{\prime}\left(-D^{t} C\right) v^{\prime}+{ }^{t} v^{\prime}\left(D^{t} A\right) v^{\prime \prime}+{ }^{t} v^{\prime \prime}\left(B^{t} C\right) v^{\prime}-{ }^{t} v^{\prime \prime}\left(B^{t} A\right) v^{\prime \prime} .
$$

As $M$ is symplectic, $D^{t} C$ is symmetric, hence only the terms $\left(D^{t} C\right)_{i i}\left(v_{i}^{\prime}\right)^{2}$ remain $\bmod 2$, but $\left(v_{i}^{\prime}\right)^{2} \equiv v_{i}^{\prime} \bmod 2$ and thus ${ }^{t} v^{\prime}\left(-D^{t} C\right) v^{\prime} \equiv\left(D^{t} C\right)_{0} v^{\prime} \bmod 2$, 
similarly $v^{\prime \prime}\left(B^{t} A\right) v^{\prime \prime} \equiv\left(B^{t} A\right)_{0} v^{\prime \prime} \bmod 2$. Next $B^{t} C \equiv I+A^{t} D \bmod 2$ and thus ${ }^{t} v^{\prime}\left(D^{t} A\right) v^{\prime \prime}+{ }^{t} v^{\prime \prime}\left(B^{t} C\right) v^{\prime} \equiv{ }^{t} v^{\prime} v^{\prime \prime} \bmod 2$. Thus we found that

$$
\begin{aligned}
& q_{\Delta}\left(M^{-1} v\right)={ }^{t} v^{\prime} v^{\prime \prime}+\left(a^{t} D-b^{t} C+\left(D^{t} C\right)_{0}\right) v^{\prime}+\left(-a^{t} B+b^{t} A+\left(B^{t} A\right)_{0}\right) v^{\prime \prime} \\
& \text { so } q_{\Delta}\left(M^{-1} v\right)=q_{M \cdot \Delta}(v), \text { as desired. }
\end{aligned}
$$

\section{A.4 Transvections}

The group $\operatorname{Sp}(2 g)$ is generated by transvections $t_{v}$, for $v \in V$, which are analogous to reflections in orthogonal groups [18, Section 6.9]. They are defined as

$$
t_{v}: V \longrightarrow V, \quad t_{v}(w):=w+E(w, v) v .
$$

It is straightforward to verify that $t_{v} \in \operatorname{Sp}(2 g)$, in fact the same formula works also for $\mathbf{Z}$ instead of $\mathbf{F}_{2}$ and then defines elements in $\operatorname{Sp}(2 g, \mathbf{Z})$. As $g t_{v} g^{-1}=t_{g(v)}$ for $g \in \operatorname{Sp}(2 g)$ and $v \in V$, the non-trivial transvections form a conjugacy class.

For $v \in V$ one verifies easily $t_{v}^{2}=1$, the identity on $V$, so $t_{v}$ is an involution, and

$$
t_{v} t_{w}=t_{w} t_{v} \quad \text { if } \quad E(v, w)=0, \quad t_{v} t_{w} t_{v}=t_{v+w} \quad \text { if } \quad E(v, w)=1
$$

for $v, w \in V$. In particular $\left(t_{v} t_{w}\right)^{2}=1$ if $E(v, w)=0$ and $\left(t_{v} t_{w}\right)^{3}=1$ if $E(v, w)=1$.

Using these Coxeter relations it is easy to establish a relation between $\operatorname{Sp}(6)$ and $W\left(E_{7}\right)$.

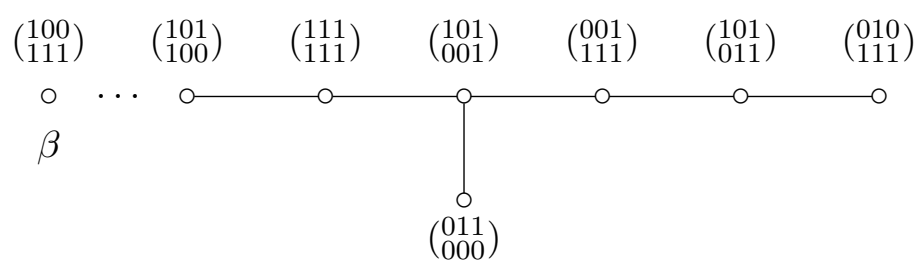

One verifies that $E(v, w)=0$ for points $v, w$ in the diagram except when they are connected by an edge (or the dotted edge), in which case $E(v, w)=1$. Thus there is a surjective homomorphism from $W\left(E_{7}\right)$ to $\operatorname{Sp}(6)$. Note that $\beta$ indicates the longest root of $E_{7}$ and from the diagram one can see that 
$\beta^{\perp}$ contains a root system of type $D_{6}$. This root system can be realized as the set of vectors $\pm\left(e_{i} \pm e_{j}\right)$ in $\mathbf{R}^{6}$, in particular, there are six mutually perpendicular vectors in $D_{6}$ (for example $e_{1}+e_{2}, e_{1}-e_{2}, e_{3}+e_{4}, \ldots, e_{5}-$ $\left.e_{6}\right)$. Therefore there are seven perpendicular roots $\beta=\beta_{1}, \ldots, \beta_{7}$ in $E_{7}$. The product of the reflections in $W\left(E_{7}\right)$ defined by these seven roots is obviously -1 on $\mathbf{R}^{7}$, thus it lies in the center of $W\left(E_{7}\right)$. The group $W\left(E_{7}\right) /\langle-1\rangle$ is simple and is isomorphic to $\operatorname{Sp}(6)$, one actually has

$$
W\left(E_{7}\right) \cong S p(6) \times\{ \pm 1\}
$$

That the image of -1 is trivial can also be checked as follows: the seven perpendicular roots map to the seven non-zero points in a Lagrangian subspace. In suitable coordinates we may assume that this subspace is $\left.L:=\left\{\begin{array}{c}a b c \\ 000\end{array}\right): a, b, c \in \mathbf{F}_{2}\right\}$ and then one verifies easily that the product of the seven transvections $t_{v}$, where $v$ runs over the non-zero elements of $L$, is the identity on $V$.

\section{A.5 The action of the transvections on the characteristics}

Let $v \in V$ and let $q$ be a quadratic form with associated bilinear form $E$ and characteristic $\Delta_{q}$. As $t_{v}$ is an involution, $q(v+w)=q(v)+q(w)+E(v, w)$ for all $v, w \in V$ and $q(a v)=a q(v)$ for $a \in \mathbf{F}_{2}$ we have

$$
\left(t_{v} \cdot q\right)(w)=q\left(t_{v}(w)\right)=q(w+E(v, w) v)=q(w)+E(v, w) q(v)+E(v, w)^{2} .
$$

Hence we get the simple rule

$$
\left(t_{v} \cdot q\right)(-)=\left\{\begin{array}{rll}
q(-) & \text { if } & q(v)=1, \\
q(-)+E(v,-) & \text { if } & q(v)=0,
\end{array} \quad \text { so } \quad t_{\left(\begin{array}{c}
v^{\prime} \\
v^{\prime \prime}
\end{array}\right)} \cdot q_{\left[\begin{array}{l}
a \\
b
\end{array}\right]}=q_{\left[\begin{array}{l}
a+v^{\prime \prime} \\
b+v^{\prime}
\end{array}\right]}\right.
$$

in case $q_{\left[\begin{array}{l}a \\ b\end{array}\right]}\left(\left(\begin{array}{c}v^{\prime} \\ v^{\prime \prime}\end{array}\right)\right)=0$.

Note that the seven points in the top row of the Dynkin diagram in Appendix A.4 are a diagram of type $A_{7}$ and that $W\left(A_{7}\right)=S_{8}$, so we do get a copy of $S_{8} \subset W\left(E_{7}\right)$, which maps, isomorphically, to a subgroup of $\operatorname{Sp}(6)$. For each of these seven points $v=\left(\begin{array}{l}a b c \\ d e f\end{array}\right) \in V$ one verifies that $q(v):=$ $a d+b e+c f=1$ where $q$ is the quadric with characteristic $\Delta_{q}=\left[\begin{array}{l}000 \\ 000\end{array}\right]$. Hence we get seven transvections in the orthogonal group $O^{+}$of $q$ which generate a subgroup isomorphic to $S_{8}$. As $O^{+}$has index 36 in $\operatorname{Sp}(6)$ it follows that $O^{+} \cong S_{8}$. 


\section{Appendix B The Heisenberg group and the theta constants}

\section{B.1 The projective representation of $\Gamma_{g}$ on $T_{g}$}

Recall that we defined $\Theta[\sigma](\tau):=\theta\left[\begin{array}{l}\sigma \\ 0\end{array}\right](2 \tau)$. Let $T_{g}$ be the $2^{g}$-dimensional vector space spanned by the holomorphic functions $\Theta[\sigma]$, with $\sigma \in \mathbf{F}_{2}^{g}$ :

$$
T_{g}:=\langle\ldots, \Theta[\sigma], \ldots\rangle_{\sigma \in \mathbf{F}_{2}^{g}} .
$$

There is a (projective, linear) action of $\Gamma_{g}$ on $T_{g}$ given by

$$
(M \cdot \Theta[\sigma])(\tau):=(\operatorname{det}(C \tau+D))^{-1 / 2} \Theta[\sigma]\left(M^{-1} \cdot \tau\right),
$$

the sign ambiguity leads to a projective, rather than a linear, representation which factors over the finite group $\Gamma_{g} / \Gamma_{g}(2,4)$. This action obviously induces usual action on the $\theta[\Delta]^{4} \in \operatorname{Sym}^{4}\left(T_{g}\right)$ as well as the $\operatorname{Sp}(2 g)$-representations $\rho_{k}$ on the Heisenberg invariants of degree $2 k$ in $\mathbf{C}[\ldots, \Theta[\sigma], \ldots]$.

To compute the action of $M \in \Gamma_{g}$ on $T_{g}$ one first considers the case that $M$ has blocks $A, \ldots, D$ with $C=0$, in which case it is straightforward to find $M \cdot \Theta[\sigma]$. Next one considers the matrix $S \in \Gamma_{g}$ given by $A=D=0$, $B=-C=I$ on the $\Theta[\sigma]$.

$$
\Theta[\sigma]\left(S^{-1} \cdot \tau\right)=\Theta[\sigma]\left(-\tau^{-1}\right)=\theta\left[\begin{array}{l}
\sigma \\
0
\end{array}\right]\left(-2 \tau^{-1}\right)=c_{\tau} \theta\left[\begin{array}{l}
0 \\
\sigma
\end{array}\right](\tau / 2),
$$

where in the last equality we use the classical transformation formula (cf. [16, II.5, Corollary]), in particular $c_{\tau}$ does not depend on $\sigma$. To express $\theta\left[{ }_{\sigma}^{0}\right](\tau / 2)$ in terms of the $\Theta[\sigma]$ 's we write each $m \in \mathbf{Z}^{g}$ uniquely as $m:=2 n+\rho$ with $\rho=\left(\rho_{1}, \ldots, \rho_{g}\right), \rho_{j} \in\{0,1\}$. Then the sum over $m \in \mathbf{Z}^{g}$ is the sum over $n \in \mathbf{Z}^{g}$ and over these $2^{g}$ elements $\rho$ :

$$
\begin{aligned}
\theta\left[\begin{array}{l}
0 \\
\sigma
\end{array}\right](\tau / 2) & =\sum_{\rho} \sum_{n \in \mathbf{Z}^{g}} \mathrm{e}^{\pi \mathrm{i}\left((2 n+\rho)(\tau / 2)^{t}(2 n+\rho)+(2 n+\rho)^{t} \sigma\right)} \\
& =\sum_{\rho} \sum_{n \in \mathbf{Z}^{g}} \mathrm{e}^{\pi \mathrm{i}(n+\rho / 2) 2 \tau^{t}(n+\rho / 2)+2 \pi \mathrm{in} n^{t} \sigma+\pi \mathrm{i} \rho^{t} \sigma},
\end{aligned}
$$

as $n^{t} \sigma \in \mathbf{Z}$ we get

$$
\Theta[\sigma]\left(S^{-1} \cdot \tau\right)=c_{\tau} \sum_{\rho}(-1)^{\rho \sigma} \Theta[\rho](\tau) .
$$

This transformation on the $\Theta[\sigma]$ 's is also known as the (finite) Fourier transform.

As $\Gamma_{g}$ is generated by the matrices with $C=0$ and $S$ (cf. [16, I.10, Lemma 15]) this allows one in principle to compute the action of any $M \in \Gamma_{g}$ on $T_{g}$. 


\section{B.2 The actions of $H_{g}$ and $\Gamma_{g}(2) / \Gamma_{g}(2,4)$}

We show that the Heisenberg group $H_{g}$ (cf. Section 2.6) and the subgroup $\Gamma_{g}(2) / \Gamma_{g}(2,4)$ of $\Gamma_{g} / \Gamma_{g}(2,4)$ act in the same way on the vector space $T_{g}$ spanned by the $\Theta[\sigma]$ 's. In particular, the Heisenberg invariants in $\mathbf{C}[\ldots, \Theta[\sigma], \ldots]$ are the $\Gamma_{g}(2) / \Gamma_{g}(2,4)$-invariants and these are thus modular forms on $\Gamma_{g}(2)$.

First of all we consider the exact sequence:

$$
\begin{array}{ccc}
0 \longrightarrow \Gamma_{g}(2) / \Gamma_{g}(2,4) & \longrightarrow \Gamma_{g} / \Gamma_{g}(2,4) \longrightarrow & \Gamma_{g} / \Gamma_{g}(2) \longrightarrow 0 \\
\cong \downarrow \phi & & \cong \downarrow \\
H_{g} / \mu_{4} & & \operatorname{Sp}(2 g)
\end{array}
$$

where, for $M=I+2 S \in \Gamma_{g}(2)$ and $S$ with blocks $A^{\prime}, B^{\prime}, C^{\prime}, D^{\prime}$, the isomorphism $\phi$ is induced by

$$
\tilde{\phi}: \Gamma_{g}(2) \longrightarrow \mathbf{F}_{2}^{g} \times \mathbf{F}_{2}^{g}, \quad M=I+2 S \longmapsto\left(\operatorname{diag} C^{\prime}, \operatorname{diag} B^{\prime}\right) \bmod 2 .
$$

It is easy to verify that $\tilde{\phi}$ is a homomorphism, its kernel consists of the $M$ with $\operatorname{diag} B^{\prime} \equiv \operatorname{diag} C^{\prime} \equiv 0 \bmod 2$, or equivalently, $\operatorname{diag}\left(I+2 A^{\prime}\right) 2{ }^{t} B^{\prime} \equiv$ $\operatorname{diag} 2 C^{\prime t}\left(1+2 D^{\prime}\right) \equiv 0 \bmod 4$, so $\operatorname{ker} \tilde{\phi}=\Gamma_{g}(2,4)$. Using $M=I+2 S$ with $A^{\prime}=D^{\prime}=0$ and with either $B^{\prime}$ diagonal and $C^{\prime}=0$ or $B^{\prime}=0$ and $C^{\prime}$ diagonal (such matrices are symplectic) one verifies the surjectivity of $\tilde{\phi}$.

Let $M \in \Gamma_{g}(2)$ be the matrix with blocks $A=D=I, \quad B=$ $\operatorname{diag}\left(2 u_{1}, \ldots, 2 u_{g}\right)$ with $u_{i} \in\{0,1\}$ and $C=0$. Then one easily verifies

$$
\begin{gathered}
(M \cdot \Theta[\sigma])(\tau)=\Theta[\sigma]\left(M^{-1} \cdot \tau\right)=\Theta[\sigma](\tau-B)=(-1)^{\sigma u} \Theta[\sigma](\tau), \\
u:=\left(u_{1}, \ldots, u_{g}\right) .
\end{gathered}
$$

In particular, $M$ acts as $(1,0, u) \in H_{g}$ on $T_{g}$. Using the identity

$$
\left(\begin{array}{cc}
I & 0 \\
C & I
\end{array}\right)=\left(\begin{array}{cc}
0 & I \\
-I & 0
\end{array}\right)\left(\begin{array}{cc}
I & -C \\
0 & I
\end{array}\right)\left(\begin{array}{cc}
0 & -I \\
I & 0
\end{array}\right)
$$

one checks that if $N \in \Gamma_{g}(2)$ is the matrix with blocks $A=D=I, B=$ $0, C=2 \operatorname{diag}\left(x_{1}, \ldots, x_{g}\right)$, then $N \cdot \Theta[\sigma]$ is $\Theta[\sigma+x]$, up to a multiplicative factor independent of $\sigma$. In particular, $N$ acts as $(1, x, 0) \in H_{g}$ on $T_{g}$. 
Summarizing, if $M \in \Gamma_{g}(2)$ and $\phi(M)=(x, u) \in \mathbf{F}_{2}^{2 g}$, then the action of $M$ and $(t, x, u) \in H_{g}$ (for any $t \in \mu_{4}$ ) on $\mathbf{P} T_{g}$ coincide. Thus the Heisenberg group action on $T_{g}$ is a lifting of the action of $\Gamma_{g}(2) / \Gamma_{g}(2,4)$ on $\mathbf{P} T_{g}$.

\section{B.3 The action of a transvection on the $\Theta[\sigma]^{\prime}$ 's}

For $v \in \mathbf{F}_{2}^{2 g}=\mathbf{Z}^{2 g} / 2 \mathbf{Z}^{2 g}, v \neq 0$, we defined the transvection $t_{v} \in \operatorname{Sp}(2 g)$ in Appendix A.4. Let $\tilde{v} \in \mathbf{Z}^{2 g}$ a vector such that $\tilde{v}$ maps to $v \in \mathbf{Z}^{2 g} / 2 \mathbf{Z}^{2 g}$ and let $t_{\tilde{v}} \in \Gamma_{g}$ be the associated transvection:

$$
t_{\tilde{v}}: \mathbf{Z}^{2 g} \longrightarrow \mathbf{Z}^{2 g}, \quad x \longmapsto x+E(x, \tilde{v}) \tilde{v}
$$

where now $E$ is the standard symplectic form on $\mathbf{Z}^{2 g}$ as in Appendix A.3. Then the image of $t_{\tilde{v}}$ in $\operatorname{Sp}(2 g)=\Gamma_{g} / \Gamma_{g}(2)$ is $t_{v}$. In particular, the linear maps $\rho_{k}\left(t_{v}\right)$ on the modular forms of weight $2 k$ on $\Gamma_{g}(2)$ are induced by the action of $t_{\tilde{v}}$ on $T_{g}$ as in Appendix B.1:

$$
t_{\tilde{v}} \cdot f=\rho_{k}\left(t_{v}\right) f, \quad f \in \mathbf{C}[\ldots, \Theta[\sigma], \ldots]_{4 k}^{H_{g}}=M_{2 k}^{\theta}\left(\Gamma_{g}(2)\right) .
$$

We give a simple description of the action of $t_{\tilde{v}}$.

Let $v=(x, u)$ and let, as in Section 2.6, $x u=x_{1} u_{1}+\cdots+x_{g} u_{g} \in \mathbf{F}_{2}$. Define $U_{v}: T_{g} \rightarrow T_{g}$ to be the action of $(1, v) \in H_{g}$ in case $x u=1$ and let it denote the action of (i, $v)$ (with $\mathrm{i}^{2}=-1$ ) in case $x u=0$. In particular, we have $U_{v}^{2}=-I$ for all $v \in V$. Next we define a linear map on $T_{g}$ by

$$
\tilde{t}_{v}: T_{g} \longrightarrow T_{g}, \quad \tilde{t}_{v}:=\frac{1-\mathrm{i}}{2}\left(U_{v}+I\right) .
$$

We will now verify that $\tilde{t}_{v}=t_{\tilde{v}}$ on $\mathbf{P} T_{g}$ and that $\tilde{t}_{v}$ induces $\rho\left(t_{v}\right)$ on the Heisenberg invariants.

\section{B.3.1 Conjugation by $\tilde{\boldsymbol{t}}_{v}$}

An important property of $\tilde{t}_{v}$ is that for all $w \in V$ there is a non-zero constant $c_{v, w} \in \mathbf{C}$ such that:

$$
\tilde{t}_{v} U_{w} \tilde{t}_{v}^{-1}=c_{v, w} U_{t_{v}(w)} .
$$

For example, if $x u=0$, then $U_{v}^{-1}=-U_{v}$ and so $\tilde{t}_{v}^{-1}=\frac{1+\mathrm{i}}{2}\left(-U_{v}+I\right)$, hence

$$
\tilde{t}_{v} U_{w} \tilde{t}_{v}^{-1}=\frac{1}{2}\left(U_{v} U_{w} U_{v}^{-1}+U_{v} U_{w}-U_{w} U_{v}+U_{w}\right)
$$


in case $E(v, w)=0$ one has $U_{v} U_{w}=U_{w} U_{v}$ hence $\tilde{t}_{v} U_{w} \tilde{t}_{v}^{-1}=U_{w}$ and if $E(v, w)=1$ one has $U_{v} U_{w}=-U_{w} U_{v}$ which is also equal to $U_{v+w}$ up to a constant, hence $\tilde{t}_{v} U_{w} \tilde{t}_{v}^{-1}=\left(U_{v} U_{w}-U_{w} U_{v}\right) / 2=c U_{v+w}$. Similarly one handles the case $x u=1$.

\section{B.3.2 The action of the lifts on $T_{g}$}

As $\Gamma_{g}(2) / \Gamma_{g}(2,4)$ is a normal subgroup of $\Gamma_{g}(1) / \Gamma_{g}(2,4)$, the group $\Gamma_{g}(1) / \Gamma_{g}(2,4)$ acts by conjugation on $\Gamma_{g}(2) / \Gamma_{g}(2,4)$. In particular one verifies easily that for $\tilde{v}, \tilde{w} \in \mathbf{Z}^{2 g}$ we have

$$
t_{\tilde{v}} t_{2 \tilde{w}} t_{\tilde{v}}^{-1}=t_{2 \tilde{w}^{\prime}}, \quad \text { with } \quad \tilde{w}^{\prime}=t_{\tilde{v}}(\tilde{w}) .
$$

In Appendix B.2 we verified that $t_{2 \tilde{w}} \in \Gamma_{g}(2) / \Gamma_{g}(2,4)$ acts as $U_{w}$ on $\mathbf{P} T_{g}$. Thus $t_{\tilde{v}} \in \operatorname{Sp}(2 g, \mathbf{Z})$ must act on $\mathbf{P}\left(T_{g}\right)$ by a linear map $S_{\tilde{v}}$ which satisfies $S_{\tilde{v}} U_{w} S_{\tilde{v}}^{-1}=U_{t_{v}(w)}$.

On the other hand, we verified in Section B.3.1 that $\tilde{t}_{v}$ has this property. Thus $S:=\tilde{t}_{v}^{-1} S_{\tilde{v}}$ satisfies $S U_{v}=U_{v} S$ for all $v \in V$. But the group generated by the $U_{v}$ acts irreducibly on $T_{g}$ (this is easy to check). Therefore, by Schur's Lemma, $S$ is a scalar multiple of the identity and thus

$$
\tilde{t}_{v}(\Theta[\sigma])=t_{\tilde{v}} \cdot \Theta[\sigma], \quad(\forall \sigma)
$$

up to a scalar independent of $\sigma$.

\section{B.3.3 The action of $\operatorname{Sp}(2 g)$ on the Heisenberg invariants}

Now we show that the action of $t_{v}$ on the Heisenberg invariants in $\mathbf{C}[\ldots, \Theta[\sigma], \ldots]$ is given by $\tilde{t}_{v}$. We already know that the action of $\tilde{t}_{v}$ is the action of $t_{v}$ up to a scalar multiple. As the degree of a Heisenberg invariant polynomial is a multiple of four, the action of $\tilde{t}_{v}$ and $i^{a} \tilde{t}_{v}$, for integer $a$, are the same on the Heisenberg invariants.

First of all we note that $\tilde{t}_{v}^{2}=-i U_{v}$ and thus $\tilde{t}_{v}^{2}$ acts as the identity on the Heisenberg invariants. Also $t_{v}$ has order two, so if $\lambda_{v} \tilde{t}_{v}$ gives the action of $t_{v}$ then $\lambda_{v}^{8}=1$. To exclude the case that $\lambda_{v}^{4}=-1$, we consider the action of $\tilde{t}_{v}$ on the $\theta[\Delta]^{4}$. This action should coincide with the one of $t_{\tilde{v}} \in \Gamma_{g}$. Choose an even characteristic $\Delta$ such that $t_{\tilde{v}} \cdot \Delta=\Delta$. As the $t_{v}$ generate the orthogonal group of the corresponding quadratic form $q_{\Delta}$, we have $\rho_{2}\left(t_{\tilde{v}}\right) \theta[\Delta]^{4}=-\theta[\Delta]^{4}$. 
Now an easy computation shows that, with the definition of $\tilde{t}_{v}$ above and the classical formula for $\theta[\Delta]^{2}$ given in 3.3 , one has

$$
\tilde{t}_{v}\left(\theta[\Delta]^{2}\right)= \pm i \theta[\Delta]^{2} \quad \text { if } \quad t_{\tilde{v}} \cdot \Delta=\Delta .
$$

Hence also $\tilde{t}_{v}\left(\theta[\Delta]^{4}\right)=-\theta[\Delta]^{4}$. Therefore $\tilde{t}_{v}$ gives the action of $t_{v}$ on the Heisenberg invariants.

\section{B.3.4 Example}

Let $g=1$ and let $v=\left(\begin{array}{l}0 \\ 1\end{array}\right) \in \mathbf{F}_{2}^{2}$. Then w.r.t. to the basis $\Theta[0], \Theta[1]$ of $T_{1}$ we have

$$
U_{v}=\mathrm{i}\left(\begin{array}{cc}
1 & 0 \\
0 & -1
\end{array}\right), \quad \tilde{t}_{v}=\frac{1-\mathrm{i}}{2}\left(U_{v}+I\right)=\left(\begin{array}{cc}
1 & 0 \\
0 & -\mathrm{i}
\end{array}\right) \quad\left(\in G L\left(T_{1}\right)\right) .
$$

From this we find the action for example on $\theta\left[\begin{array}{l}0 \\ 0\end{array}\right]^{4}$ :

$$
\tilde{t}_{v}\left(\theta\left[\begin{array}{l}
0 \\
0
\end{array}\right]^{4}\right)=\tilde{t}_{v}\left(\Theta[0]^{2}+\Theta[1]^{2}\right)^{2}=\left(\Theta[0]^{2}-\Theta[1]^{2}\right)^{2}=\theta\left[\begin{array}{l}
0 \\
1
\end{array}\right]^{4} .
$$

Let $\tilde{v}=\left(\begin{array}{l}0 \\ 1\end{array}\right) \in \mathbf{Z}^{2}$, then

$$
t_{\tilde{v}}=t_{\left(\begin{array}{l}
0 \\
1
\end{array}\right)}=\left(\begin{array}{ll}
1 & 1 \\
0 & 1
\end{array}\right)=T \quad\left(\in \Gamma_{1}\right)
$$

and thus we recover the action of $T$ on $\theta\left[\begin{array}{l}0 \\ 0\end{array}\right]^{4}$.

Note that if $v=(0, u) \in \mathbf{F}_{2}^{g} \times \mathbf{F}_{2}^{g}$ then $U_{v}$ is diagonal, and so is $\tilde{t}_{v}$ (which corresponds to an element with $C=0, A=D=I$ in $\operatorname{Sp}(2 g, \mathbf{Z})$ ), so we can compute the action in a particularly simple (and fast!) manner.

\section{References}

[1] J. H. Conway, R. T. Curtis, S. P. Norton, R. A. Parker and R. A. Wilson, Atlas of finite groups, Oxford University Press, Eynsham, 1985.

[2] W. Bosma, J. Cannon and C. Playoust, The Magma algebra system. I, The user language, J. Symb. Comput. 24 (1997), 235-265.

[3] S. L. Cacciatori, F. Dalla Piazza, Two loop superstring amplitudes and $S_{6}$ representation, Lett. Math. Phys. 83 (2008), 127-138.

[4] S. L. Cacciatori, F. Dalla Piazza and B. van Geemen, Modular forms and three loop superstring amplitudes, Nucl. Phys. B800 (2008), 565590, arXiv:0801.2543. 
[5] S. L. Cacciatori, F. Dalla Piazza and B. van Geemen, Genus four superstring measures, Lett. Math. Phys. 85 (2008), 185-193, arXiv:0804.0457.

[6] E. D'Hoker and D. H. Phong, Two-loop superstrings IV: the cosmological constant and modular forms, Nucl. Phys. B 639 (2002), 129-181.

[7] E. D'Hoker and D. H. Phong, Asyzygies, modular forms, and the superstring measure I, Nucl. Phys. B $\mathbf{7 1 0}$ (2005), 58-82.

[8] E. D'Hoker and D. H. Phong, Asyzygies, modular forms, and the superstring measure II, Nucl. Phys. B 710 (2005), 83-116.

[9] J. S. Frame, The classes and representations of the group of 27 lines and 28 bitangents, Ann. Math. Pura Appl. 32 (1951), 83-119.

[10] J. S. Frame, Some characters of orthogonal groups over the field of two elements, in Procceedings of the Second International Conference on the Theory of Groups, Lecture Notes in Math., vol. 372, Springer, Berlin, 1974, 298-314.

[11] J. S. Frame, Degree polynomials for the orthogonal groups over GF(2), C. R. Math. Rep. Acad. Sci. Canada 2 (1980), 253-258.

[12] B. van Geemen, Siegel modular forms vanishing on the moduli space of curves, Invent. Math. 78 (1984), 329-349.

[13] B. van Geemen and G. van der Geer, Kummer varieties and moduli spaces of abelian varieties Am. J. Math. 108 (1986), 615-642.

[14] S. Grushevsky, Superstring scattering amplitudes in higher genus, Commun. Math. Phys. 287 (2009), 749-767, arXiv:0803.3469.

[15] B. Heim, Miyawaki's $F_{12}$ spinor L-function conjecture, arXiv:0712.1286.

[16] J. Igusa, Theta functions, Springer-Verlag, Berlin, New York, 1972.

[17] J. Igusa, Schottky's invariant and quadratic forms, in "International Christoffel Symposium Proceedings", eds. P. L. Butzer and F. Fehér, Birkhäuser, 1981, 352-362.

[18] N. Jacobson, Basic algebra I, W. H. Freeman and Company, San Francisco, 1974.

[19] A. Morozov, NSR Superstring Measures Revisited, JHEP 0805:0806, (2008), arXiv:0804.3167v1.

[20] I. Miyawaki, Numerical examples of Siegel cusp forms of degree 3 and their zeta-functions, Mem. Fac. Sci. Kyushu Univ. Ser. A 46 (1992), 307-339.

[21] M. Oura, private communication. 
[22] M. Oura and R. Salvati Manni, On the image of code polynomials under theta map, J. Math. Kyoto Univ. 48(4) (2008), 895-906, arXiv:0803.4389.

[23] B. Runge, On Siegel modular forms, part I, J. Reine Angew. Math. 436 (1993), 57-85.

[24] B. Runge, On Siegel modular forms, part II, Nagoya Math. J. 138 (1995), 179-197.

[25] R. Salvati Manni, Modular varieties with level 2 theta structure, Amer. J. Math. 116 (1994), 1489-1511.

[26] R. Salvati Manni, Remarks on superstring amplitudes in higher genus, Nucl. Phys. B801(1-2) (2008), 163-173. arXiv:0804.0512.

[27] S. Tsuyumine, On Siegel modular forms of degree three, Amer. J. Math. 108 (1986), 755-862. 